\title{
Visual stimulation with blue wavelength light drives V1 effectively eliminating stray light contamination during two-photon calcium imaging
}

\author{
Tatiana Kuznetsova ${ }^{1, *}$, Kamil Antos ${ }^{1}$, Evgenya Malinina, Stylianos Papaioannou, Paolo Medini \\ Department of Integrative Medical Biology, Umeå University, SE-901 87 Umeå, Sweden
}

\section{A R T I C L E I N F O}

\section{Keywords:}

In vivo population $\mathrm{Ca}^{2+}$ imaging

Light contamination

Visual stimulation

Mouse primary visual cortex

Visually-evoked potentials

3D acousto-optic two-photon imaging

\begin{abstract}
A B S T R A C T
Background: Brain visual circuits are often studied in vivo by imaging $\mathrm{Ca}^{2+}$ indicators with green-shifted emission spectra. Polychromatic white visual stimuli have a spectrum that partially overlaps indicators' emission spectra, resulting in significant contamination of calcium signals.

New method: To overcome light contamination problems we choose blue visual stimuli, having a spectral composition not overlapping with $\mathrm{Ca}^{2+}$ indicators emission spectrum. To compare visual responsiveness to blue and white stimuli we used electrophysiology (visual evoked potentials -VEPs) and 3D acousto-optic two-photon (2P) population $\mathrm{Ca}^{2+}$ imaging in mouse primary visual cortex (V1).

Results: VEPs in response to blue and white stimuli had comparable peak amplitudes and latencies. $\mathrm{Ca}^{2+}$ imaging in a Thy1 GP4.3 line revealed that the populations of neurons responding to blue and white stimuli were largely overlapping, that their responses had similar amplitudes, and that functional response properties such as orientation and direction selectivities were also comparable.

Comparison with existing methods: Masking or shielding the microscope are often used to minimize the contamination of $\mathrm{Ca}^{2+}$ signal by white light, but they are time consuming, bulky and thus can limit experimental design, particularly in the more and more frequently used awake set-up. Blue stimuli not interfering with imaging allow to omit shielding.

Conclusions: Together, our results show that the selected blue light stimuli evoke responses comparable to those evoked by white stimuli in mouse V1. This will make complex designs of imaging experiments in behavioral setups easier, and facilitate the combination of $\mathrm{Ca}^{2+}$ imaging with electrophysiology and optogenetics.
\end{abstract}

\section{Introduction}

$\mathrm{Ca}^{2+}$ imaging is widely used to monitor neuronal responses to different stimuli and conditions at population, axonal, dendritic and dendritic spine levels both in vitro and in vivo (Dana et al., 2019; Grienberger and Konnerth, 2012). Somatic and axonal calcium transients accompany neuron's spike output, whereas dendritic $\mathrm{Ca}^{2+}$ signals can identify local and global dendritic activations driven by synaptic input as well as by back-propagating action potentials (Ali and Kwan, 2019). Combination of $\mathrm{Ca}^{2+}$ imaging with optogenetic tools permits to manipulate the activity of specific neuronal populations with high spatiotemporal precision, allowing to dissect the functional organization of neural circuits in different model organisms (e.g. flies, worms, fish and mammals, spanning from mice to non-human primates) and in various brain regions (Carrillo-Reid et al., 2017; Galvan et al., 2017; Jiao et al., 2018; Shipley et al., 2014; Simpson and Looger, 2018).

Changes in intracellular $\mathrm{Ca}^{2+}$ concentration are estimated by changes in fluorescence of $\mathrm{Ca}^{2+}$ indicators, emitting the light of certain wavelengths if activated by excitation light when bound to calcium. Currently genetically-encoded calcium indicators (GECIs) are the most used, as they can also be stably expressed in transgenic animals (Dana et al., 2014, 2018). The most commonly used GECIs - GCaMPs - have calmodulin as a $\mathrm{Ca}^{2+}$ sensor and cpEGFP (circularly permuted enhanced green fluorescent protein) as a reporter. cpEGFP has a green emission spectrum peaking at around $515 \mathrm{~nm}$, when GCaMP is bound to $\mathrm{Ca}^{2+}$. Recently developed red-shifted GECI - such as jRGECO1a, jRCaMP1a and $\mathrm{b}$ have emission spectra peaking at around $600 \mathrm{~nm}$ (Dana et al., 2016, 2018).

Regardless of GECI used, the detection of activity-driven changes in its fluorescence can be hindered by light contamination (Leinweber et al., 2014). Indeed, the spectra of ambient light (e.g. from monitor

\footnotetext{
* Corresponding authors.

E-mail addresses: tatiana.kuznetsova@helsinki.fi (T. Kuznetsova), paolo.medini@umu.se (P. Medini).

1 Equal contribution.
} 


\section{Nomenclature}

3D three-dimensional

2P two-photon

V1 primary visual cortex

VEP visually-evoked potential

LFP Local field potential

i.m. intramuscular (injection)

AOD acousto-optic deflectors

PMT photomultiplier tube

ROI region of interest

SD standard deviation

cPI chromatic preference index

OI orientation index

DI direction index

GECIs genetically-encoded calcium indicators

EGFP enhanced green fluorescent protein

CRT cathode-ray tube

IOI Intrinsic optical imaging

ERG electroretinogram

screens) overlaps with the emission spectra of GECIs. For example, the white light of CRT (cathode-ray tube) screens, often used for visual stimuli presentation, is formed by a combination of red, green, and blue primary colors, spreading between 380 and $730 \mathrm{~nm}$ (Fig. 1A). Light contamination is particularly problematic in the case of visual stimulation, used to explore for example the visual cortex (both primary and associative), a classical model to investigate the function and plasticity of cortical microcircuits in vivo (Yang et al., 2018). In addition, visual stimuli are used in awake set ups as visual cues (Goard et al., 2016; Hwang et al., 2019), in visually guided eye movement tasks (Itokazu et al., 2018) and in virtual reality systems when the functions of (pre) motor (Leinweber et al., 2017) and associative (Krumin et al., 2018) cortical networks are investigated. Without accurate microscope and objective shielding stray light contamination can cause a two-fold increase of the basal background brightness (see for example Fig. 2 in (Leinweber et al., 2014)), largely reducing the dynamic range of the response.

A common way to bypass this problem is to mask the gap between objective and imaged brain area with opaque material (Leinweber et al., 2014). This limits the experiment to an optical approach, excluding (or rendering problematic) simultaneous electrophysiological recording, optogenetic manipulation of an area outside the imaged one, or drug application. Alternatively, one can shield the objective by placing black curtains (often close to the preparation) between mice eye and craniotomy. This solution however might be bulky or mechanically undoable, preventing or creating problems in open frame behavioral experiments, and sometimes might not be sufficient.

Another approach to overcome the light contamination is the synchronization of the screen's or projector's (that are generating the visual signal) output to the turnaround time of the laser scanning electronics (when the signal is not collected (Leinweber et al., 2014; Reiff et al., 2010)). This solution requires however engineering for synchronization for each setup, use of LED screens and might present limitations in some types of scanning (like random access, acousto-optic scanning used here).

The third approach, considered here, is to substitute the white light (used for visual stimuli and causing the light contamination) with light that has the spectrum spectrally separated from GECI emission. Taking into account the current existence of "green" and "red" GECI, blue light $(\sim 395 / 400-495 / 500 \mathrm{~nm})$ could be a good option (Fig. 1A).

However, although visual stimulation with blue light (400-500 nm) has been used during in vivo imaging (Lecoq et al., 2014), it has not been systematically studied whether visual stimulation with blue light, selected to minimize the contamination of the recorded "green" calcium indicator signal (500-540 nm), evokes visual responses similar to the ones triggered by the white light in primary visual cortex (V1) of experimental rodents. Rodents, in particularly mice, are primarily nocturnal animals with dichromatic color vision and rod-dominated retinas (Huberman and Niell, 2011). Here we tested whether visual responsiveness and functional response properties of visual V1 neurons (e.g. orientation and directional selectivity) are similar in response to white and blue stimuli. It is possible that the blue light could trigger a weaker response, since the wavelength composition of blue light covers a smaller part of mice spectral sensitivity function, as obtained from electroretinogram (ERG) and behavioral tests (Jacobs et al., 1991, 2004; Rocha et al., 2016). On another hand, blue and white lights should activate two main pigments with similar absorption spectra in retinal photoreceptors: rhodopsin in rods and the "green" long/middle (L/M) wave-sensitive opsin in cones (but very weakly shortwave/ultraviolet (S/UV)-sensitive opsin), as predicted by their spectral sensitivity functions (Peirson et al., 2018).

To address this question, we recorded VEPs and performed 3D acousto-optic $2 \mathrm{P}$ population $\mathrm{Ca}^{2+}$ imaging (that allows to monitor the activity of chosen multiple individual neurons) in $\mathrm{V} 1 \mathrm{of} \mathrm{a} \mathrm{Ca}^{2+}$ reporter mice line (Thy1-GP4.3)) during the presentation of white/black and blue/black gratings, and measured if the responses to this two colortypes of gratings are significantly different.

\section{Materials and methods}

\subsection{Animal strain and housing}

For LFP experiments young adult (1-2 months) C57BL/ $6 \mathrm{~J}$ male wild type mice were used. For 2-photon $\mathrm{Ca}^{2+}$ imaging of neuronal population young adult (1-3 months) Thy1-GCaMP6s mice (C57BL/6 J-Tg(Thy1GCaMP6s)GP4.3Dkim/J; The Jackson Laboratory, N 024275) were used (Dana et al., 2014). Mice were housed in Umeå Center for Comparative Biology's (UCCB) and maintained in a $12 \mathrm{~h}$ light/dark cycle, with food and water available ad libitum. At the end of the experiment animals were sacrificed by cervical dislocation. All procedures were done according to the Ethical permit from the Swedish Ethical Committee for Northern Sweden.

\subsection{In-vivo extracellular electrophysiology}

\subsubsection{Mouse preparation and surgery}

Animals were anesthetized with isoflurane (4\% for induction; $1.5-2.5 \%$ during surgery). To reduce the inflammatory response and brain edema Dexamethasone $(0.7 \mathrm{mg} / \mathrm{kg}$, i.m.; $60-100 \mu \mathrm{l}$ of $0.2 \mathrm{mg} / \mathrm{ml}$ in saline) was administered before the surgery. To protect the cornea from dehydration, we applied artificial tears (VISCOTEARS $2 \mathrm{mg} / \mathrm{g}$; FASS) on the eyes and covered them with $\emptyset 6 \mathrm{~mm}$ cover glass. Body temperature during the experiments was constantly monitored with a rectal thermocouple probe and maintained at $37^{\circ} \mathrm{C}$ with a feedbackcontrolled heating blanket (using ATC-2000 Animal Temperature Controller).

After removing the skin and periosteum, a metal head-plate was glued to the bone with Superglue in the hemisphere opposite to the planned craniotomy. Then a recording bath from dental cement (Paladur) was made at the borders of the skull. The attachment of the headplate and the bath was reinforced by tissue glue (3 M Vetbond). The bath allowed to keep the cortical surface always covered by physiological solution $(0.9 \% \mathrm{NaCl})$ and to place the ground electrode. After cement polymerization the bone above the V1 was carefully thinned by drilling and a small rectangular craniotomy was cut with a surgical blade ca $3 \mathrm{~mm}$ mediolateral from lambda (above the binocular portion of V1). 

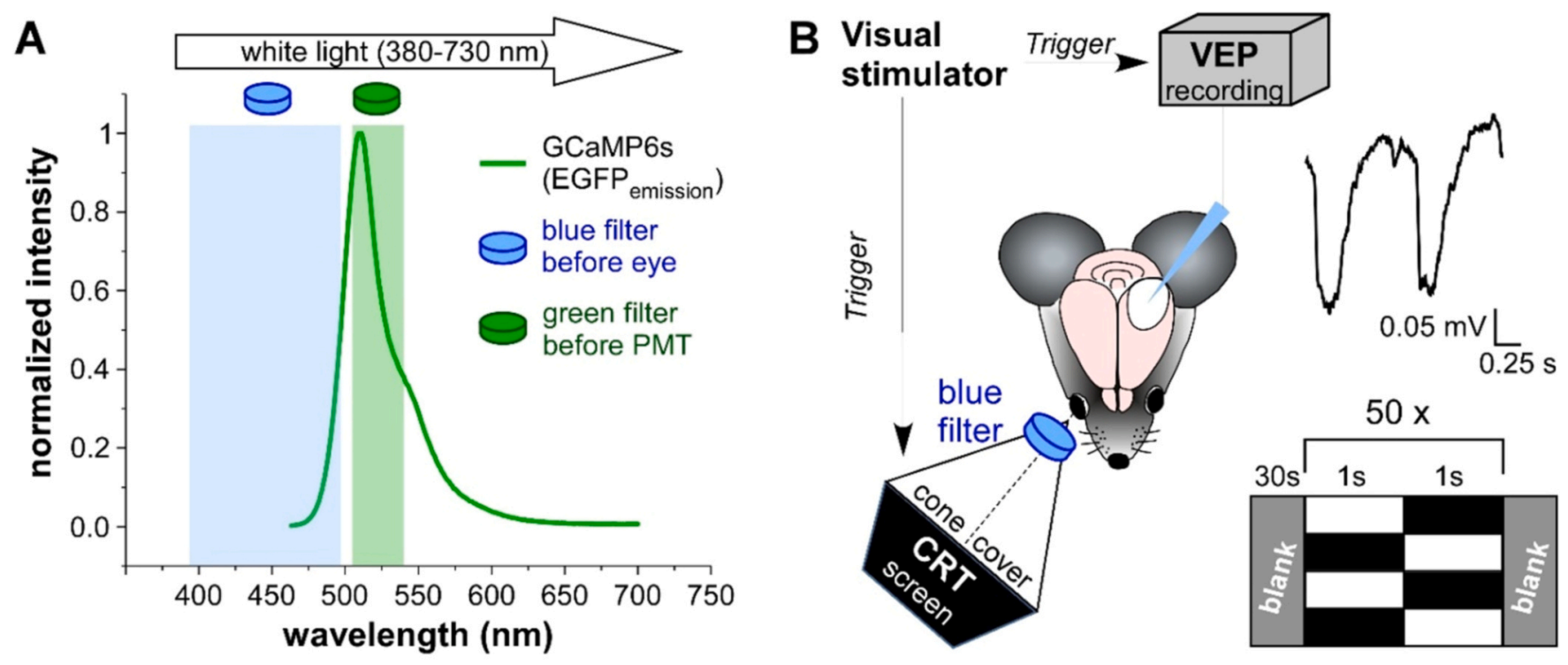

C

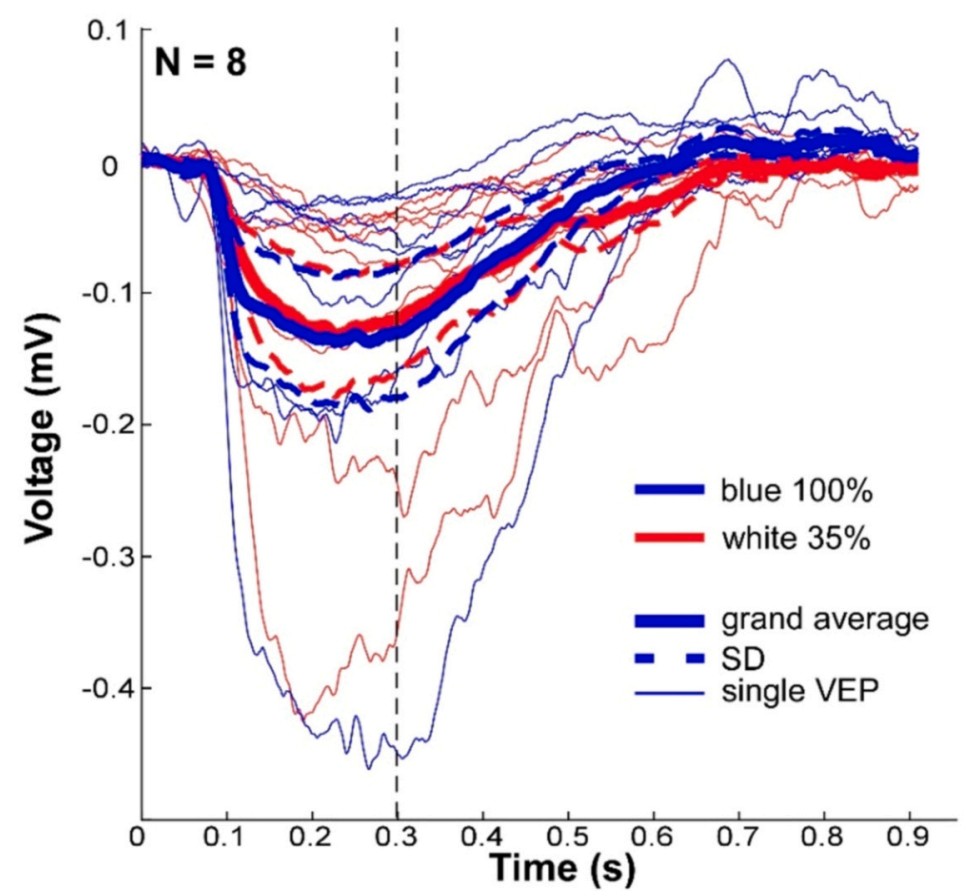

D

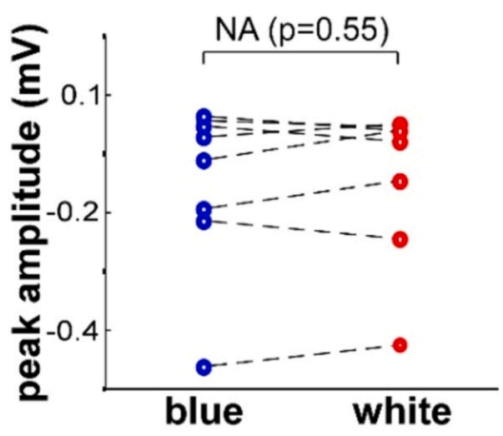

E

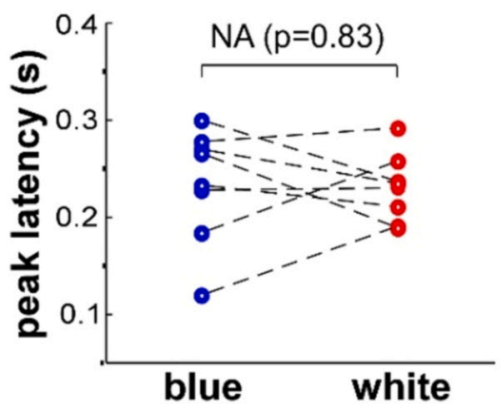

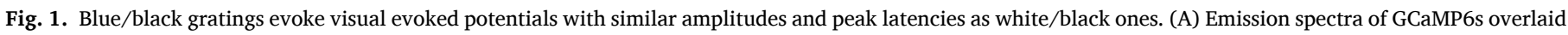

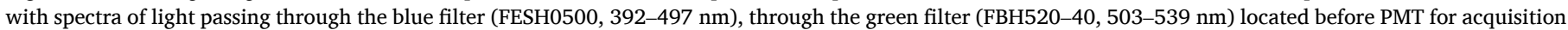

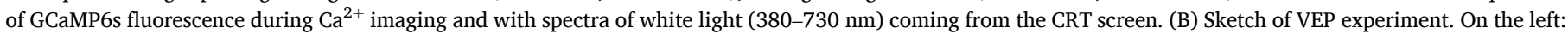

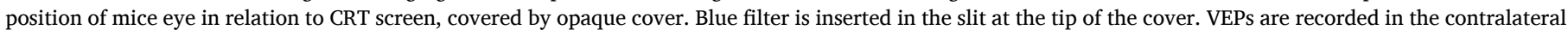

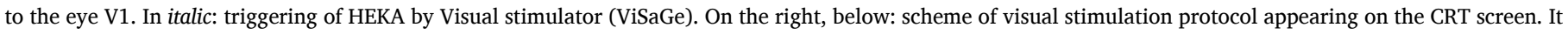

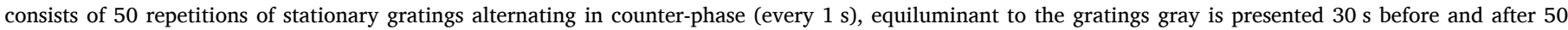

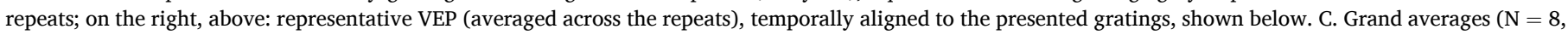

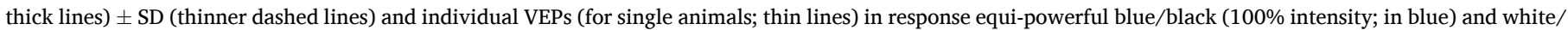

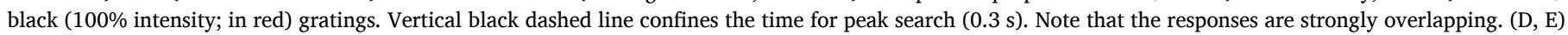

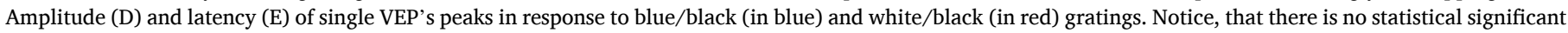

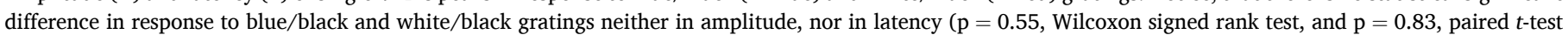
0.84 , respectively; $\mathrm{N}=8$ ).

\subsubsection{Local field potentials (LFP) recordings}

A glass micropipette (1-2 M $\Omega$; $\varnothing$ ca $10 \mu \mathrm{m})$ filled with saline $(0.9 \%$ $\mathrm{NaCl})$ was positioned at a cortical depth of $200 \mu \mathrm{m}$. LFP were recorded using an ELC-03XS amplifier (NPI Electronic; Germany) and acquired via HEKA LIH $8+8$ data acquisition interface and PATCHMASTER software (HEKA; Germany). Signals were amplified (x1000), bandpass- filtered $(0.1-100 \mathrm{~Hz})$ and digitized $(1 \mathrm{kHz})$. LFP recordings were triggered by the onset of visual stimulus (reversal of the stationary gratings, see below) appearing on the CRT screen and controlled by a stimulus generator (ViSaGe from Cambridge Research Systems Ltd; UK; see Fig. 1B, left). Each recording (1 sweep) lasted $1.91 \mathrm{~s}$ (grating pattern reversed at 0 and $1 \mathrm{~s}$ ) and 50 such sweeps were recorded within a series 
A

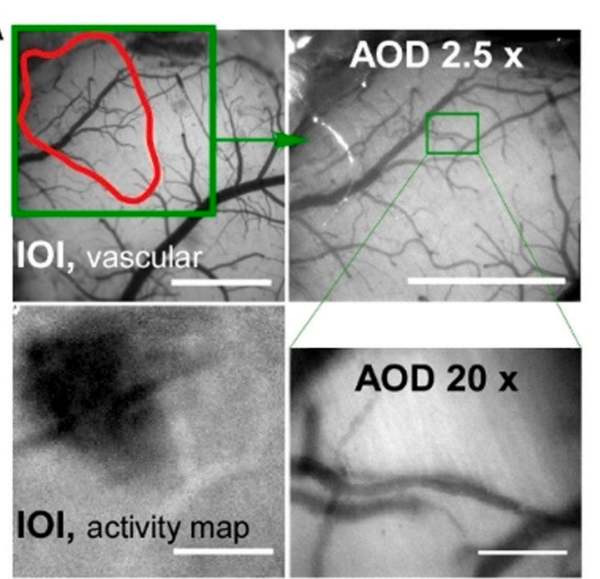

B

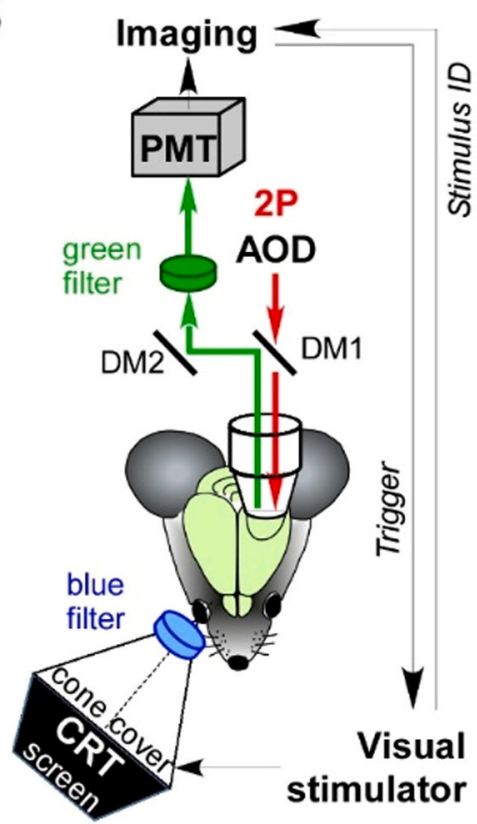

E

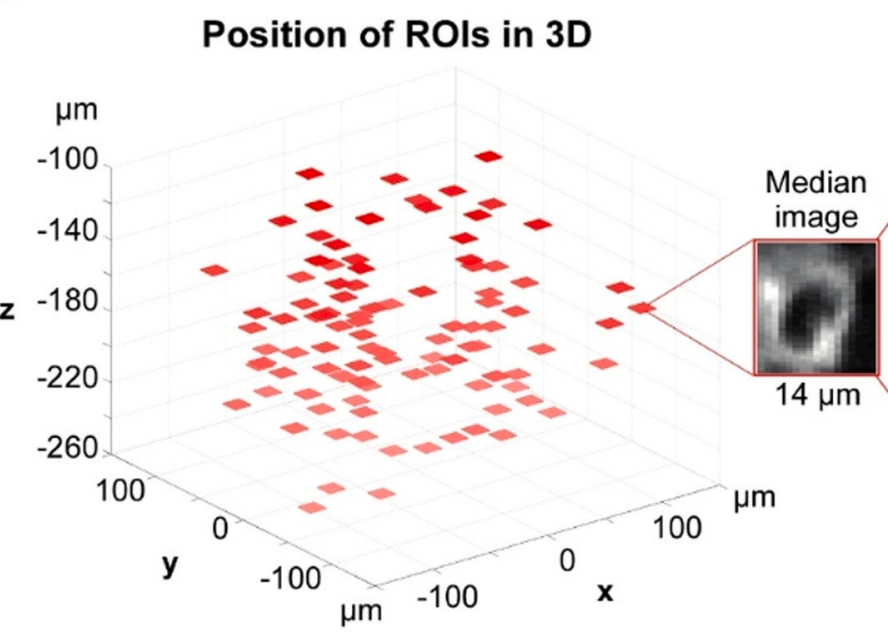

D
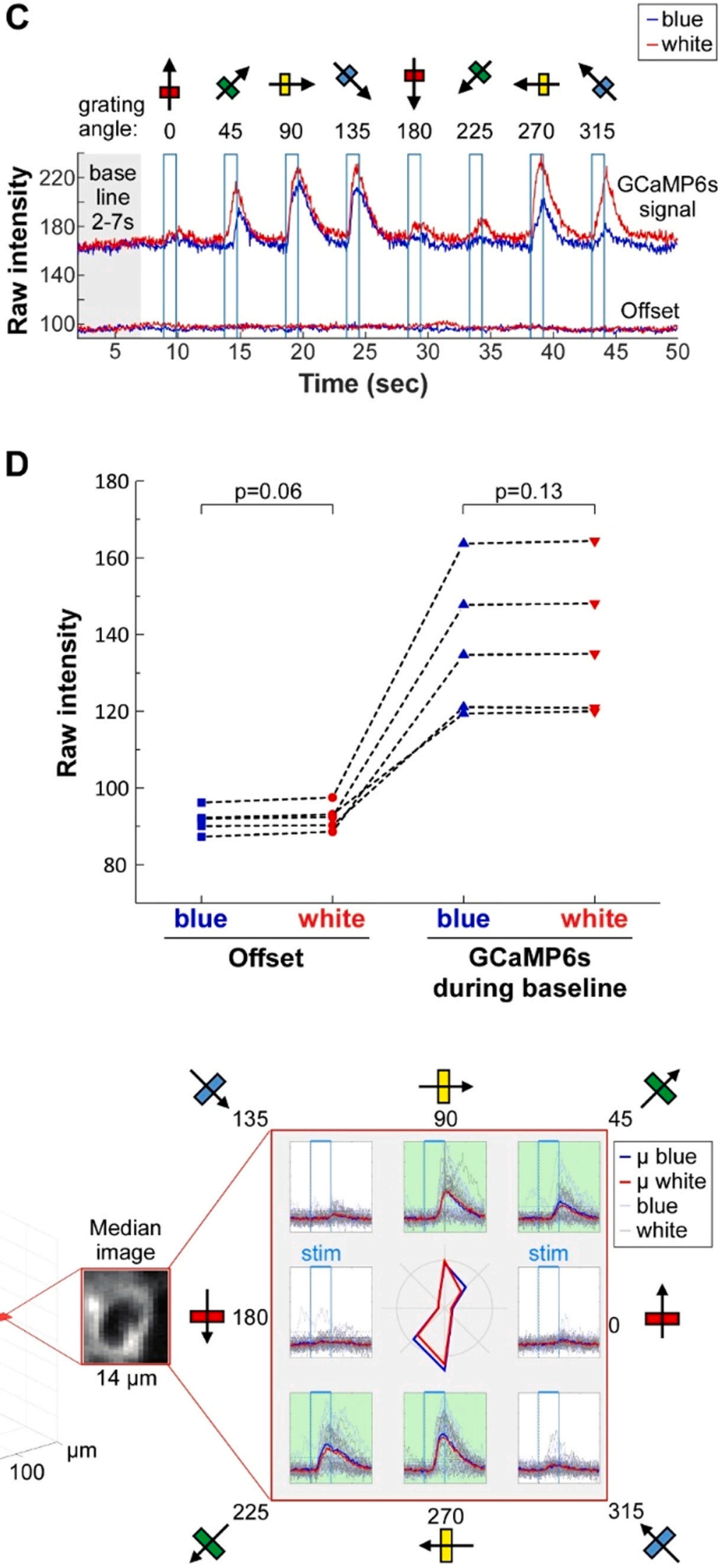
Fig. 2. Experimental design and processing of $\mathrm{Ca}^{2+}$ imaging. (A) Selection of the region for $\mathrm{Ca}^{2+}$ imaging. Example obtained by optical imager of vascular image (upper left) overlaid with outlined in red visual activity map obtained by IOI (lower left). The same area in camera mode under AOD setup at low magnification (2.5x; upper right) with the region selected for $\mathrm{Ca}^{2+}$ imaging (outlined in green). The same region at higher magnification (20x, lower right). The scale bar for IOI images and AOD 2.5x is $1 \mathrm{~mm}$ and for AOD 20x $-100 \mu \mathrm{m}$. (B) Sketch of $2 \mathrm{P} \mathrm{Ca}^{2+}$ imaging using AOD setup, performed in V1 of Thy1-GCaMP6s mice during visual stimulation, presented on the CRT screen. In italic: triggering and timing control of the system. DM1 and DM2 - dichroic mirrors 1 and 2. (C) Example of time courses of (1, above) raw intensities of GCaMP6s fluorescence plotted for individual ROI during two single acquisition trials and (2, below) offset values in representative experiment - intensities averaged over all ROIs while laser shutter was closed. Intensities during blue/black (in blue) or white/black (in red) gratings presentation. Note that "blue" and "white" offset values overlap along entire time course; as well do the raw intensities of GCaMP6s fluorescence during the baseline period (2-7 s; highlighted in grey) used for $\mathbf{F}_{\mathbf{0}}$ calculation, suggesting comparable level of light contamination between two stimulation types. Stimulation periods (1 $\mathrm{s}$ each) are outlined with blue rectangles; directions of drifting gratings are indicated above. (D) Mean offset values (first 2 columns) and mean raw GCaMP6s fluorescence values during baseline period (II in C; last 2 columns) upon blue/black (in blue) or white/black (in red) gratings presentation. Values are averaged across all ROIs, all trials and time (of entire acquisition for offset or period within 2-7 s for raw GCaMP6s fluorescence) within 1 experiment (1 animal). Squares, circles or triangles represent single animals $(\mathrm{N}=5$ ). (E) Left: example of acquired neurons located in 3D space. In the middle - median ROI image of representative cell, created across the data frames within the experiment. Right: responses $\left(\Delta \mathrm{F} / \mathrm{F}_{0}\right)$ of this cell to blue/black (in blue) or white/black (mean - in read, single recordings - in gray) gratings plotted separately for each of 8 directions; thick lines: mean $\Delta \mathrm{F} / \mathrm{F}_{0}(\mu)$ averaged over the trials ( $\mathrm{n}=21$ and 17 for blue and white respectively); thin: $\Delta \mathrm{F} / \mathrm{F}_{0}$ of individual trials; in the middle - polar plots of the responses evoked by blue/black (in blue) or white/black (in red) gratings. Stim - time of the grating presentation.

(see Fig. 1B, right).

Series with white/black and blue/black grating presentations were recorded in a random order in different animals.

\subsubsection{Visual stimulation during $L F P$ recording}

We presented stimuli on a calibrated video monitor system (ViSaGe MKII Stimulus Generator, Cambridge Research Systems Ltd, CRT monitor HP P1230, refresh rate $140 \mathrm{~Hz}$, color temperature $9300 \mathrm{~K}$ ), controlled by custom-made scripts in Matlab. The CRT screen was lightshielded by the opaque cone with a round slit on its end for blue filter insertion. This slit was positioned in close vicinity to the mouse eye (contralateral to the craniotomy), with the CRT screen located at ca $40 \mathrm{~cm}$ from the eye. Visual stimuli (see Fig. 1B, lower right) were stationary gratings alternating in counter-phase (horizontally oriented; with squared spatial profile, spatial frequency 0.05 cycles/degree, and temporal frequency $0.5 \mathrm{~Hz}$ ). White/black or blue/black gratings with equal output power (ca $3 \mu \mathrm{W}$, measured by silicon power meter Thorlabs PM100D, at $40 \mathrm{~cm}$ distance from the screen, on the level of the slit) were presented. To reach similar output power levels for these two visual stimuli we used the following configurations: When white/black gratings were used, the intensity of the white on the CRT screen was set to $35 \%$ and the filter was removed from the slit in the cone. In case of blue/black gratings intensity of white was set to $100 \%$ and a blue filter (FESH0500, Thorlabs, bandpass: $392-497 \mathrm{~nm}$ ) was inserted into the slit.

\subsubsection{LFP data processing}

LFP responses to each pattern reversal within a series (100 reversals, 50 sweeps - 2 reversals/sweep) were averaged so to measure VEP responses for both color-types of gratings. The first $40 \mathrm{~ms}$ of each series were considered as a baseline, as there is not even retinal ganglion cell activation during this time window (Mazzaro et al., 2016), and no synaptic activation of layer $4 \mathrm{~V} 1$ neurons (Medini, 2011). The average voltage reading during $0-40 \mathrm{~ms}$ window was subtracted from each averaged trace (allowing for VEP peak search and alignment of the different series for grand average calculation). This was done in a custom-made software, written in MatLab. VEP peaks were searched within 300 ms post-stimulus onset (pattern reversal) in OriginPro 2017 software; peak amplitudes and latencies were determined. For grand average calculations, VEPs of individual animals $(\mathrm{N}=8)$ in response to white/black or blue/black gratings were averaged and grand mean \pm standard deviation (SD) were plotted (see Fig. 1C).

\subsection{D Acousto-optic 2-photon $\mathrm{Ca}^{2+}$ imaging of neuronal populations}

\subsubsection{Mouse preparation and surgery}

Surgical anesthesia and animal preparation were done as described for LFP experiments. Part of the skin was cut out, the periosteum was removed and a circular titanium head post was attached to the skull using superglue, tissue glue and dental cement with binocular V1 in the center ( $3 \mathrm{~mm}$ mediolateral from lambda). This head post was used later to create a bath chamber, filled with saline warmed to body temperature (for IOI) or with ultrasound gel (for $\mathrm{Ca}^{2+}$ imaging). In the middle of this bath, a circular craniotomy (ca $4 \mathrm{~mm}$ in diameter) was made. To cover the craniotomy, a round glass coverslip ( $\varnothing 5 \mathrm{~mm}$ ) was glued to the bone with a tiny amount of superglue. Since isoflurane (1-1.5\%) anesthesia can affect the patterns of $\mathrm{Ca}^{2+}$ activity in mouse V1 (Goltstein et al., 2015), for the recording of visually-evoked activity we switched to a lighter anesthesia regimen: with chlorprothixene $(1 \mathrm{mg} / \mathrm{kg}, 60-100 \mu \mathrm{l}$ of $0.3 \mathrm{mg} / \mathrm{ml}$ in saline, i.m.; (Nauhaus et al., 2012)) and $0.5 \%$ isoflurane that was gradually reduced after the surgery (Juavinett et al., 2017).

\subsubsection{Intrinsic optical imaging (IOI)}

We used IOI to identify the location of V1 relative to the blood vessel pattern. For images acquisition an Optical Imager 3001 system (Optical Imaging Inc, Rehovot, Israel) was used, equipped with: (1) HAL 100 illuminator with quartz collector (423000-9901-000) as a light source with DC power supply (TPR3020S, Atten instruments), (2) a macroscope consisting of 2 tandem Nikon lenses (NIKKOR $50 \mathrm{~mm} \mathrm{f} / 1.2$ and AF DCNIKKOR $135 \mathrm{~mm} \mathrm{f} / 2 \mathrm{D}$ ) attached to $25 \mathrm{~Hz}$ CCD-camera (A-1000 m/D, Adimec, 237127) and (3) Vdaq software for data acquisition. The Optical imaging Vdaq software triggered the Visual stimuli generator (ViSaGe) via custom-written routine in Matlab.

A "green" vascular image was captured while illuminating the cortex with $546 \pm 30 \mathrm{~nm}$ light (bandpass filter 91933, Thorlabs) with the pia surface in focus (see Fig. 2A, upper left). To obtain activity maps (see Fig. 2A lower left), images were acquired with monochromatic $630 \mathrm{~nm} \pm 10 \mathrm{~nm}$ light filter (Thorlabs), defocused ca 500-600 $\mu \mathrm{m}$ below the pial surface, while the lenses diaphragm was open. Image size was approximately $2.7 \times 2.7 \mathrm{~mm}$ and $996 \times 996$ pixels; $3 \times 3$ spatial online binning was applied. During $10 \mathrm{~s}$ of stimulation 50 data frames were acquired (200 ms each, consisting of 5 camera frames). During IOI drifting gratings of 0 or 90 degree (sine waveform, spatial frequency 0.03 cycles/degree, drift velocity 1 cycle/sec; $100 \%$ contrast) or equiluminant blanks were displayed on the screen. Stimuli (0/90 degree grating and the blank) were presented in random order with $15 \mathrm{~s}$ of inter-stimulus interval. The protocol was repeated 10 times.

After a pixel by pixel subtraction of the first 10 frames from subsequent ones, activity maps were obtained by dividing the images of cortex, stimulated with the gratings, by the average blank image of the unstimulated cortex (to correct for uneven illumination (Grinvald et al., 1999)). The obtained maps of visual response to 0 or 90 degree gratings were largely overlapping (Fig. S2.1). The visually active part of V1 was found by overlaying the activity map with vasculature image acquired before (see Fig. 2A upper left, visually active area is outlined in red). The activity map was used to guide subsequent $2 \mathrm{P} \mathrm{Ca}^{2+}$ imaging. The vascular pattern of the active area was used as a landmark for alignment during $2 \mathrm{P}$ set up using a low magnification objective (Zeiss 2.5x air objective, $0.06 \mathrm{NA}$; see Fig. 2A upper right) with help of the microscope 
camera system. Part of these area was chosen for $\mathrm{Ca}^{2+}$ imaging, performed under higher (20x) magnification (see Fig. 2A lower right).

\subsection{3. $\mathrm{Ca}^{2+}$ imaging}

$2 \mathrm{P} \mathrm{Ca}^{2+}$ imaging of local populations was performed using a $2 \mathrm{P}$ microscope with acousto-optic deflectors (AOD, Femtonics femto 3D Omega), providing random access acquisition of multiple cell somata in 3D (Szalay et al., 2016). Data acquisition was controlled by the MES software (Femtonics; for setup sketch see Fig. 2B). The laser source was a Ti-Saphire laser (Tsunami 3941C-25XP femtosecond laser), running at $875 \mathrm{~nm}$, pumped with Millennia eV 25S (Spectra-Physics). The objective used for $\mathrm{Ca}^{2+}$ imaging was a 20x water immersion lens with high numerical aperture (NA 1.0) and $2 \mathrm{~mm}$ working distance (Olympus XLUMPLFLN-W). As an immersion fluid ultrasound gel Aquasonic 100 (Parker) was used. To minimize light contamination and the differences in offset value while presenting gratings of different colors, the gap between objective and the bath was masked with opaque plasticine. The power used to excite GCaMP6s was on average $84 \mathrm{~mW}$ (range: 73-106). The GCaMP6s expressing somata (with surrounding background areas) were selected from a 3D stack that was acquired before recording within the $\mathrm{L} 2 / 3$ ( $~ 80-260 \mu \mathrm{m}$ under the pia). We used a chessboard scanning approach ((Szalay et al., 2016); see Fig. 2E, left) where for each chosen cell a $14 \times 14 \mu \mathrm{m}$ region of interest (ROI, with $0.7-0.8 \mu \mathrm{m}$ pixel size) was acquired. The scanning speed was on average $21.24 \mathrm{~Hz}$ (range: 18.6-24.3; depending on the number of ROIs). To estimate amount of light contamination (offset) during given stimulation type, the acquisition was repeated with the laser shutter closed.

\subsubsection{Visual stimulation during $\mathrm{Ca}^{2+}$ imaging}

As during LFP recordings, the CRT screen was shielded by an opaque cone with a slit for insertion of the blue filter and placed at $40 \mathrm{~cm}$ from mouse eye, centered with respect to pupil projection at an angle of circa 40-45 degree from the vertical meridian. Visual stimuli were drifting blue/black or white/black gratings (with sine waveform, spatial frequency 0.03 cycles/degree, drift velocity 1 cycle/s). As it was described in the Section 2.2.3 ("Visual stimulation during LFP recording") white/ black and blue/black gratings had equal output powers and the "blue" stimulus was achieved as $100 \%$ white on the CRT screen + blue filter (see Fig. 2B). During each stimulus trial, sinusoidal drifting gratings of 8 different directions $(0,45,90,135,180,225,270,315,360$ degree angle) were presented. Stimulation with each grating direction lasted $1 \mathrm{~s}$, with a minimum $3 \mathrm{~s}$ of blank period before (see Fig. 2C). We used "compass" coordinates, in which horizontal gratings moving upward are considered to be moving at 0 degree, and angles increase in a clockwise manner (Mazurek et al., 2014). Somata were acquired for $50 \mathrm{~s}$ (one trial duration). Before, after stimulus trial and in-between gratings equi-luminant blanks were presented. The acquisition trial was repeated at least 10 times for each stimulation type (white/black or blue/black gratings). After $5 \mathrm{~s}$ of spontaneous activity recording, the MES $2 \mathrm{P}$ data acquisition system triggered the whole visual stimulation cycle ( 8 differently oriented grating presentations + blanks). The time of grating presentation (managed by ViSaGe system) and the grating direction ID were always recorded in MES software (via analog input to align and associate the data frames with grating ID (see Fig. 2B,C)).

A higher background contamination during white/black gratings presentation due to white light component could have caused a higher offset, thereby higher baseline fluorescence of GCaMP6s $\left(F_{0}\right)$ and, consequently, spurious similarity of the calcium transients between two conditions, even in case of weaker response to blue/black gratings. We thus took the countermeasures and masked the gap between objective and the bath with opaque plasticine. We then recorded the signal from selected cellular ROIs while the laser shutter was closed. In these conditions, obtained signal (offset) should be only due to the dark noise and noise from the electronics. Fig. $2 \mathrm{C}$ shows that the representative "offset" time courses recorded in these configuration during blue/black and white/black gratings presentation strongly overlapped. Averaged over the time of acquisition "offset" values were slightly bigger during white/ black gratins presentation, but the difference did not reach statistical significance ("offset": $91.55 \pm 1.46$ and $92.39 \pm 1.52$; $\mathrm{p}=0.06$ for blue and white gratings, respectively, Wilcoxon signed rank test; Fig. 2D, first 2 columns). More importantly, the raw values of acquisition time course section, used for the "baseline" fluorescence $\left(\mathrm{F}_{0}\right)$ calculation $(2-7 \mathrm{~s}$, during presentation of blank), averaged for all cells and trials within each experiment (referred to as "mean raw baseline"), were not significantly different during the presentation of blue and white blanks: $137.33 \pm 8.36$ and $137.66 \pm 8.44$, respectively; Wilcoxon signed rank test, $\mathrm{p}=0.13$; Fig. 2D (last 2 columns).

\subsubsection{Analysis of $\mathrm{Ca}^{2+}$ imaging}

Analyses were performed in MatLab. First, offset values (due to residual light contamination from ambient light and visual stimulation) were calculated for each experiment and stimulation type (blue/black or white/black gratings) as mean intensity over all pixels of each ROI over time course of acquisition trial with the laser shutter closed. This value was subtracted from all pixels from each ROI across entire acquisition duration. Later a circular standard mask was placed over the cytoplasmic region of the cell within each ROI. Masks' positions were adjusted manually for each cell and each acquisition trial to correspond to the median image created across the data frames within that trial. The adjustment was meant to correct at least partially for brain motion artifacts. For each data frame, the intensities of the pixels within and outside the mask were averaged, providing the values $\mathbf{F}_{\text {cell_measured }}(\mathbf{t})$ and $\mathbf{F}_{\text {neuropil ( } t \text { ) }}$ respectively (for the cell and neuropil). The fluorescent signal of the cell body was calculated as:

$\mathrm{F}_{\text {cell_true (t) }}=\mathrm{F}_{\text {cell_measured }(\mathrm{t})}-\mathrm{r} \times \mathrm{F}_{\text {neuropil ( }} \mathrm{t}$

Although for optimal neuropil correction $\mathrm{r}$ should be adjusted depending on depth, we have chosen to use $r=0.7$ across preparation in order to compare our data with those obtained with conventional mirror-based scanning systems with the same GECI (GCaMP6s) we had (either virally delivered or stably expressed in the same mouse strain we used here) and with a similar visual stimulation protocol, where the same $r$ value was used for all neurons (Chen et al., 2013; Dana et al., 2014). Moreover, $r$ has been reported to vary within a limited range within L2/3 (of ca 0.1, e.g. in (Kerlin et al., 2010)). The fluorescence time course of the cell body was plotted and aligned to the onset of individual grating presentation.

The baseline fluorescence $\left(\mathbf{F}_{\mathbf{0}}\right)$ was estimated as the intensity corresponding to $20 \%$ of the cumulative distribution function of $\mathrm{F}_{\text {cell }}$ true ( $\mathrm{t}$ ) within $2^{\mathrm{d}}-7$ th $\mathrm{s}$ of acquisition. $\Delta \mathrm{F} / \mathbf{F}_{\mathbf{0}}$ was calculated as:

$\Delta \mathrm{F} / \mathrm{F}_{0}=\left(\mathrm{F}_{\text {cell_true }(\mathrm{t})}-\mathrm{F}_{0}\right) / \mathrm{F}_{0}$

In order to transfer the $\Delta \mathrm{F} / \mathrm{F}_{0}$ time courses of each acquisition trial into polar coordinates, they were split into 8 segments, each for a single direction of drifting gratings with $1 \mathrm{~s}$ of pre-stimulus, just before stimulus onset, and $3 \mathrm{~s}$ after it (see Fig. 2E, right). For each direction the blank value was defined as $\Delta \mathrm{F} / \mathrm{F}_{0}$ averaged over $1 \mathrm{~s}$ of pre-stimulus. Then, mean blank value across single "blue" or "white" acquisition trials segments (recorded during blue/black or white/black grating presentation, respectively) was calculated for a given cell and direction and subtracted from $\Delta \mathrm{F} / \mathrm{F}_{0}$ values of single trial segments to bring the prestimulus to zero. Single and mean "blue" and "white" $\Delta \mathrm{F} / \mathrm{F}_{0}\left(\boldsymbol{\mu} \Delta \mathbf{F} / \mathbf{F}_{0}\right)$ time courses were then plotted for each cell and direction (see Fig. $2 \mathrm{E}$, right).

For each trial and direction, visual responses of individual cells were defined as the maximum $\Delta \mathrm{F} / \mathrm{F}_{0}$ within $2 \mathrm{~s}$, starting from stimulus onset. For a neuron to be qualified as visually responsive two conditions had to be simultaneously satisfied: (1) its visual responses for each orientation had to be significantly different from the corresponding blank value using a paired statistic (Wilcoxon signed rank test, $\mathrm{p}<0.01$ ); (2) its max $\mu \Delta \mathrm{F} / \mathrm{F}_{\mathbf{0}}$ within $2 \mathrm{~s}$ from the stimulus onset (referred to as mean 
response or response amplitude) had to be above mean blank (prestimulus $\left.\mu \Delta \mathrm{F} / \mathrm{F}_{0}\right)+2 \mathrm{SD}$.

The orientation (OI) and direction (DI) indexes were calculated for visually responsive cells (Mazurek et al., 2014). Preferred orientation ( $\mathbf{R}_{\text {pref_ori }}$ ) was determined for blue/black or white/black gratings as orientation with largest magnitude of the response among the 4 orientations. Magnitude of each orientation was calculated as sum of max $\mu \Delta \mathrm{F} / \mathrm{F}_{0}$ of two corresponding opposite directions. The orientation orthogonal to $\mathrm{R}_{\text {pref_ori }}\left(\mathbf{R}_{\text {orth_ori }}\right)$ was defined as $R_{\text {pref_ori }}+90^{\circ}$. OI was computed as:

$\mathrm{OI}=\left(\mathrm{R}_{\text {pref_ori }}-\mathrm{R}_{\text {orth_ori }}\right) / \mathrm{R}_{\text {pref_ori }}$

Preferred direction ( $\mathbf{R}_{\text {pref }}$ ) for blue/black or white/black gratings was determined as direction with largest max $\mu \Delta \mathrm{F} / \mathrm{F}_{0}$ among the 8 gratings directions presented. Null direction $\left(\mathbf{R}_{\text {null }}\right)$ was defined as direction opposite to $\mathrm{R}_{\text {pref }}\left(R_{\text {null }}=R_{\text {pref }}+180^{\circ}\right)$. DI was calculated as:

$\mathrm{DI}=\mathrm{R}_{\text {pref }}-\mathrm{R}_{\text {null }} / \mathrm{R}_{\text {pref }}$,

To quantify relative responsiveness of neurons towards blue/black versus white/black gratings, we defined a chromatic preference index (cPI, (Aihara et al., 2017; Tan et al., 2015)) as.

$\mathrm{cPI}=\mu \Delta \mathrm{F} / \mathrm{F}_{0}$ (blue, max) $-\mu \Delta \mathrm{F} / \mathrm{F}_{0}$ (white, max) $/ \mu \Delta \mathrm{F} / \mathrm{F}_{0 \text { (blue, max) }}+\mu \Delta \mathrm{F} / \mathrm{F}_{0}$ (white, $\max$ ),

where $\boldsymbol{\mu} \Delta \mathbf{F} / \mathbf{F}_{\mathbf{0}}$ (blue, max) and $\boldsymbol{\mu} \Delta \mathbf{F} / \mathbf{F}_{\mathbf{0}}$ (white, max) are the mean responses of an individual cell to preferred direction evoked by blue/black and white/black gratings, respectively. cPIs of 1 and -1 indicate complete blue/black and white/black dominances, respectively. Neurons giving identical responses to blue/black and white/black gratings have a preference index equal to " 0 “.

\subsection{Statistical analysis}

The statistical analysis was done in Origin 2017 and in MatLab. To compare two paired groups with low sample size or groups that were significantly different from normally distributed populations we used non-parametrical paired Wilcoxon signed-rank test. Normality was examined using Shapiro-Wilks test. To compare two paired normallydistributed groups paired $t$-test was used. Multiple matched groups (percentage of responsive to white, blue, or both gratings) were compared with the non-parametric Friedman test. Two independent groups (mean response of cells responsive to only blue or white gratings vs those of cells responsive to both) were compared with nonparametrical Mann-Whitney test. Cumulative distributions (of OIs and DIs) were compared by Kolmogorov-Smirnov test. The relationship between OI or DI of cell in response to blue and white gratings was estimated by linear fit function slope (k) and Pearson correlation coefficient (r).

\section{Results}

We first selected a "blue filter" transmitting a light spectrum (392-497 nm) from the CRT screen that would not overlap with the acquired GCaMP6s emission spectrum, band-passed by the "green filter" (503-539 nm, FBH520-40, Thorlabs) before the microscope's photomultiplier tube (PMT) (see Figs. 1A and 2B). The "blue" filter was inserted into the slit at the tip of an opaque frustum CRT-screen cover (see Figs. 1B and 2B) in front of mouse eye. To compare the responses to blue and white, the intensities of the stimuli were adjusted to equal powers at eye level (ca. $3 \mu \mathrm{W}$ ): CRT intensity was set to $100 \%$ for blue and $35 \%$ for white (see Section 2.2.3 - "Visual stimulation during LFP recording").
3.1. Blue/black and white/black gratings trigger visual evoked potentials with similar amplitudes and peak latencies

To estimate the effectiveness of the blue light in driving mouse V1 activation compared to the white light, we first recorded VEPs in layer $2 / 3$ in response to blue/black or white/black stationary gratings alternating in counter-phase presented to the contralateral eye of anesthetized mice, adapted to the ambient light level of the set-up used for physiology experiments (Fig. 1B; for details see the Section 2.2.3 - "Visual stimulation during LFP recording"). Blue/black gratings evoked similar responses compared to the ones evoked by white/black gratings, as shown by the largely overlapping VEP grand average traces recorded in the two conditions ( $\mathrm{N}=8$ mice; Fig. $1 \mathrm{C}$ ). A paired comparison of the peak amplitudes and latencies of VEPs, determined within $0.3 \mathrm{~s}$ from stimulus onset, showed that these parameters were not significantly different (blue vs white, peak amplitudes: $-0.148 \pm 0.051$ vs $-0.140 \pm 0.047 \mathrm{mV}$; peak latencies: $0.235 \pm 0.021$ vs $0.230 \pm 0.012 \mathrm{~s}$; Wilcoxon signed rank tests, $\mathrm{p}=0.55$ and paired $t$-test, $\mathrm{p}=0.83$, respectively; Fig. 1D,E). Thus, local synaptic inputs into the V1 network in response to white and blue light stimulations, as represented by VEPs, had similar magnitudes and reached the network with comparable latencies.

\subsection{Blue/black and white/black gratings activate two strongly overlapping neuronal subnetworks to a similar extent, as revealed by $2 P$ population $\mathrm{Ca}^{2+}$ imaging}

We next addressed the same question, at supra-threshold (spike output) level using 3D 2P population $\mathrm{Ca}^{2+}$ imaging in layer $2 / 3$ of V1, performed with an AOD 2P microscope (see the Section 2.3.3. - " $\mathrm{Ca}^{2+}$ imaging"), technique which allows fast network monitoring with single cell-resolution. We compared the cellular response to blue/black and white/black gratings drifting in one of eight different directions presented to the contralateral eye of lightly-anesthetized Thy-1-GCaMP6s mice (see the Section 2.3. - 3D Acousto-optic 2-photon $\mathrm{Ca}^{2+}$ imaging of neuronal populations). See also entire Fig. 2.

After defining the cells with a significant visual response to at least one direction of the gratings (see the Section 2.3.5. - "Analysis of $\mathrm{Ca}^{2+}$ imaging"), we calculated the percentage of the neurons responding to either stimuli in each experiment ( $\mathrm{N}=5$ mice; $81-100$ neurons were recorded per animal, and 452 in total). Blue/black and white/black drifting gratings activated a similar percentage of V1 neurons (Fig. 3A; $\mathrm{p}=0.88$, Wilcoxon signed rank test; on average: $13.9 \pm 3.6 \%$ and $13.5 \pm 3 \%$, respectively). Besides, the fraction of cells, responsive to both color-types of gratings, was also comparable to abovementioned values (Fig. 3A; Friedman test; $\mathrm{p}=0.12$; on average: $10.9 \pm 2.4 \%$ ), suggesting that two populations - responsive for blue and for white strongly overlap. The fraction of responsive neurons in $\%$ matches the one reported in the original description of the reporter line (Dana et al., 2014). Indeed, across all responsive cells $(\mathrm{N}=75)$ the largest portion (66.7\%, 50 cells) responded to both color-types of gratings (Fig. 3B), meaning that blue and white activated largely the same neuronal subnetwork. Smaller populations of cells were responsive only to the blue/black $(18.7 \%, 14$ cells) or only to the white/black $(14.7 \%, 11$ cells) gratings (Figs. 3B and S3.1). A closer examination of these cells revealed that their mean responses ( $\max \mu \Delta \mathrm{F} / \mathrm{F}_{0}$ values) in the preferred direction were lower than those of the cells responding to both color-types of gratings (in response to blue: $0.63 \pm 0.07$ for cells responsive to blue only versus $1.07 \pm 0.10$ for cells responsive to both, $\mathrm{p}=0.03$, Mann-Whitney test (Fig. S3.1C); in response to white: $0.56 \pm 0.05$ for cells responding to white only versus $1.05 \pm 0.09$ for cells responding to both, $\mathrm{p}=0.006$, Mann-Whitney test (Fig. S3.1D)). These amplitude values are also in lines with those reported for this transgenic line (Dana et al., 2014).

We next tested whether blue and white visual stimuli, beyond activating a common V1 subnetwork, triggered similar responses. So, we 
A

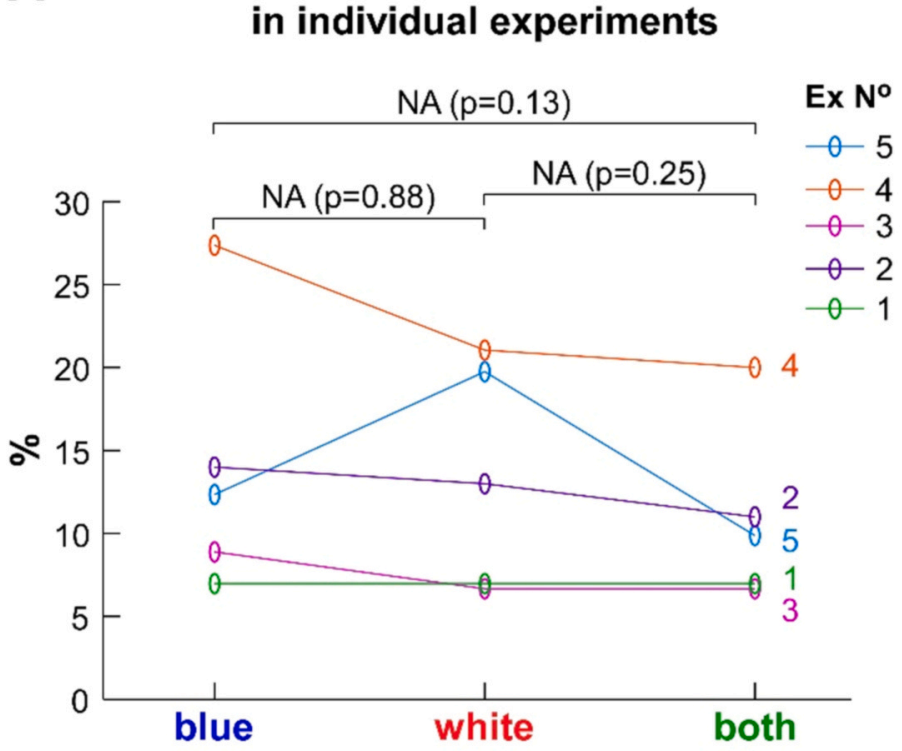

B

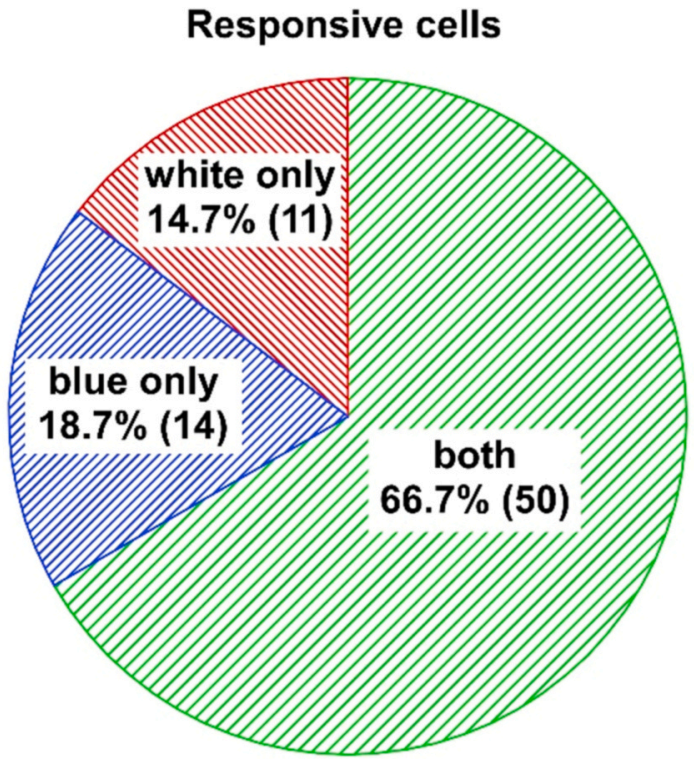

C

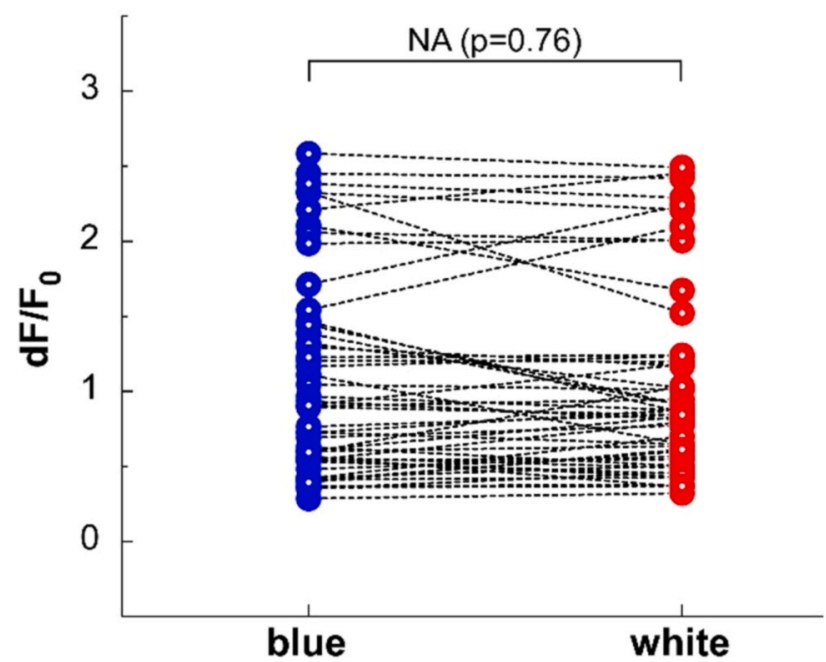

Amplitude in preferred direction Cells responsive to both

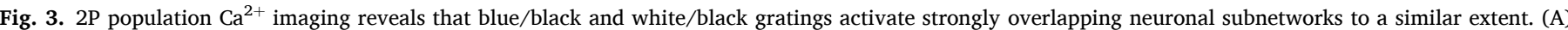

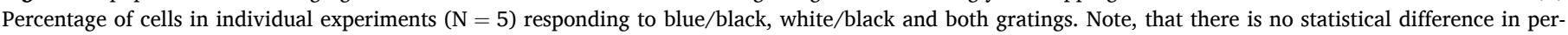

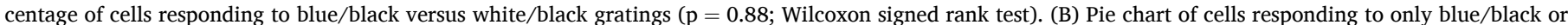

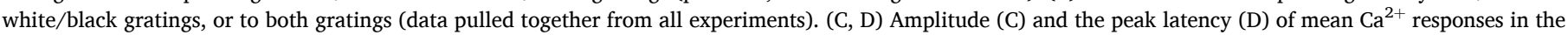

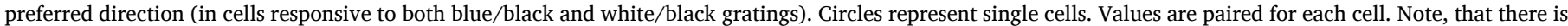

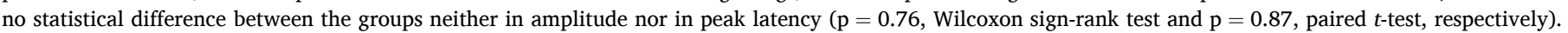

analyzed the amplitude ( $=$ mean response $=\max \mu \Delta \mathrm{F} / \mathrm{F}_{0}$ values) and the peak latency of the visually-driven $\mathrm{Ca}^{2+}$ responses in cells responsive to both stimuli. Both response parameters upon stimulation with white/ black vs blue/black gratings drifting in the cells preferred orientation were similar at the single-cell level (Fig. 3C, D; $\mathrm{n}=50$ cells (from 5 mice); on average, peak amplitudes: $1.05 \pm 0.09$ vs $1.07 \pm 0.10$, and peak latencies: $1.03 \pm 0.03$ vs $1.04 \pm 0.03 \mathrm{~s} ; \mathrm{p}=0.76$, Wilcoxon signed rank test; and $\mathrm{p}=0.87$, paired $t$-test, respectively). The same was true in all the other (non-preferred) directions: the differences between response to blue and to white in their amplitudes and latency's values, calculated separately for each cell and each direction, were clearly distributed around zero (see Fig. S3.2). Noticeably, the temporal profiles of $\mathrm{Ca}^{2+}$ transients visually-evoked by blue and white were strikingly similar (Fig. S3.3). Thus, the degree of visual responsiveness to white/ black and blue/black gratings was similar for both the preferred and non-preferred orientations for major part V1 network responsive to both stimuli.

To estimate the color preference of the whole neuronal population, we looked at the distribution of the chromatic preference index (cPI; see the Section 2.3.5. - "Analysis of $\mathrm{Ca}^{2+}$ imaging"). cPIs distributions (1) of the cells responding to both blue and white gratings $(\mathrm{N}=50$; Fig. S3.4A) as well as (2) of all responsive cells ( $\mathrm{N}=75$; Fig. S3.4B) were normally distributed (Shapiro-Wilks tests, $\mathrm{p}=0.41$ and $\mathrm{p}=0.59$, respectively) and had mean values approximating $0 \quad(-0.002 \pm 0.018$ and $-0.003 \pm 0.02$, respectively), suggesting that on the level of neuronal population there was no color preference. 
3.3. Orientation and direction selectivities are comparable upon blue/ black and white/black gratings presentation

Taking into account that direction and orientation selectivities are important functional response properties of V1 neurons, we next examined whether these parameters are different upon blue and white visual stimuli presentation. Polar plots obtained from the responses evoked by blue/black or white/black gratings in cells activated by both stimuli looked very similar (Fig. 4A, B). Polar plots of individual cells with robust responses to these two colors (with mean $\mathrm{Ca}^{2+}$ responses in preferred direction above the median response of the population of responsive to both stimuli cells) were overlapping as well (Fig. 4C). In line with that, the majority of the cells activated by both colors shared the same preferred orientation and direction in response to blue and to white, as the difference in these variables in degrees, between the two colors was close to zero (Fig. S4.1).

We further looked at the degree of preference (described by selectivity indexes: OI / DI). A paired analysis of the orientation indexes (OIs) of individual cells did not reveal any significant difference (Fig. 4D; mean OIs for blue and white: $0.67 \pm 0.03$ vs $0.66 \pm 0.03$, respectively; Wilcoxon signed rank test; $\mathrm{p}=0.57$ ). In line with that, we failed to find any statistically-significant difference between the cumulative distributions of $\mathrm{OI}$ in response to these two color-types of gratings (Fig. 4E; Kolmogorov-Smirnov test, $\mathrm{p}=0.68$ ).

On the contrary we observed strong positive relationship between $\mathrm{OI}$ of cell in response to blue/black and OI in response to white/black gratings (linear fit function slope, $\mathrm{k}=0.76$ ), in complement to strong correlation $(\mathrm{r}=0.84)$ (Fig. 4F).

The directional selectivity of responses visually-evoked by white/ black and blue/black stimuli was also similar. Indeed, we found neither difference in the paired analysis of the direction indexes (DIs) in response to white and blue color-types of gratings (Fig. 4G; mean DIs: $0.34 \pm 0.03$ vs $0.32 \pm 0.03$, respectively; Wilcoxon signed rank test, $\mathrm{p}=0.35$ ), nor in the cumulative distributions of this parameter (Fig. $4 \mathrm{H}$, Kolmogorov-Smirnov test, $\mathrm{p}=0.95$ ) but instead we observed a positive relationship $(\mathrm{k}=0.71)$, as well as correlation $(\mathrm{r}=0.7)$ between them (Fig. 4I). Of note, direction selectivity was less pronounced than orientation selectivity (mean OI $=0.67$ and 0.66 ; while mean $\mathrm{DI}=0.32$ and 0.34 for blue and white gratings, respectively), in agreement with previous reports (Niell and Stryker, 2008).

\subsection{Split test on responses to stimulation with blue and white lights}

We next estimated the internal consistency of visual responses to blue and white stimulation. We restricted this analysis to the 50 neurons responding to both colors of light and we took in consideration the amplitude of the response in the cell's preferred orientation. Trials were subdivided in odd and even trials, and new averages were calculated for the odd and even trials for each chromatic condition (whiteODD, whiteEVEN, blueODD, blueEVEN) for each cell. Fig. 5A shows, that blueODD and blueEVEN responses are slightly different, but this difference is significant (Wilcoxon Signed Rank tests, $p=0.0013$ ). Such a difference is not observed neither in the white pairs nor in the "allwhite" vs "all-blue" responses. In line with that, the blueODD-blueEVEN differences are not exactly centered at zero, whereas the whiteODDwhiteEVEN ones and "all blue"-"all-white" ones are (Fig. 5B). We next quantified how the found "all-blue"-"all-white" differences compare the whiteODD-whiteEVEN (and blueODD-blueEVEN) differences. The (ODD-EVEN) difference for blue was significantly distinct from that for white (Fig. 5B; one-way RM ANOVA, $\mathrm{p}=0.002$; followed by posthoc Tukey: $\mathrm{p}=0.002$ ).

\subsection{Convolution of monitor output and retinal spectral sensitivity}

In order to estimate quantitatively to which extent the similarity of the cortical responses could be due to similar degree of retinal recruitment by the two stimuli, we computed the convolution between the spectral sensitivity function of the retina, as assessed by ERG, and the monitor spectral output. The monitor spectral power was calculated from the Planck's law for $\mathrm{t}=9300 \mathrm{~K}$ (standard CRT white color, as of specifications). First, we calculated the spectral power of the monitor's white light with $35 \%$ intensity (White 35\% in Fig. 6A), as was set during our experiments. Then, we calculated the spectral power of the monitor filtered with the blue filter (bandpass $392-497 \mathrm{~nm}$ ) that we used to create blue light (Blue 100\% in Fig. 6A). The relative spectral sensitivities of the mouse $\boldsymbol{S}, \boldsymbol{M}$ opsin as well as the total sensitivity ( $\boldsymbol{T}$ or Total) was derived from ERG (Rocha et al., 2016). Using the above information, we simulated our experimental conditions by convolving M_White $\mathrm{x} \mathrm{T}$ and M_Blue $\mathrm{x} T$ and comparing the responses (see Fig. 6B).

Responses in V1 were simulated as the integral of the convolution curves because the average $\mathrm{V} 1$ response integrates the retinal response for each wavelength within the corresponding spectra. In such a case the integral of the blue trace (M_Blue x T) is $94.23 \%$ of the white (M_White $x$ $\mathrm{T})$. This explains why our actual experimental responses from both conditions (35\% intensity of white light vs $100 \%$ of band-passed blue light) had virtually the same amplitude (see Fig. 6C).

Also, by running separate convolutions of the monitor configurations with the $\mathrm{S}$ and $\mathrm{M}$ spectra we were able to calculate the relative contribution of $\mathrm{M}$ and $\mathrm{S}$ cones to the total response for the different configurations (see Fig. 6D, left and right, respectively). Specifically, we found that in "white monitor configuration" the relative contribution of $S$ was $12 \%$ while of the $\mathrm{M}$ was $88 \%$. Respectively during the "blue monitor configuration" S contributed $18 \%$ of the total response while M $82 \%$.

These results suggest that the similarity of cortical responsiveness is largely attributable to a comparable integrated retinal response to the two different chromatic stimuli. Moreover, the green opsins (either the $\mathrm{M}$ cone or the rods' opsin - having very similar spectra) are the ones similarly recruited by both stimuli configuration.

\section{Discussion}

White light $(\sim 380-730 \mathrm{~nm})$ contaminates the signal in network calcium imaging experiments (up to 2-fold increase in background intensity, see Fig. 2 in Leinweber et al. (2014)). Here, to minimize such contamination during $\mathrm{Ca}^{2+}$ imaging, we used blue light $(\sim$ 392-497 nm), spectrally distinct from the "green" GCaMP6s signal (filtered before PMTs: 503-539 nm; Fig. 1A). We expected weaker visual responses, since blue light comprises a smaller portion of visible spectrum absorption of both the rods' rhodopsin (peak: $498 \mathrm{~nm}$ ) and cones' "green" (M/L) opsin (peak: $508 \mathrm{~nm}$ ), than the white one does.

Our data suggests instead that stimulation with blue light results in similar local synaptic inputs (as assessed by LFP) and triggers a comparable spike network output (measured optically by random access 3D two-photon calcium imaging). Of relevance, fundamental functional response properties such as orientation and direction selectivity - a hallmark of V1 neurons (Huberman and Niell, 2011), especially in layer 2/3 (Hübener, 2003; Niell and Stryker, 2008)- were also similar.

4.1. Comparable physiological response in V1 L2/3 to blue and white visual stimuli as due by similar integrated retinal activation

A comparison of V1 responses to equi-powerful stimuli with partially overlapping spectra (white embraced the entire spectra of blue) has not been done before. However, comparison of V1 responses to two spectrally distinct colors, that match two main peaks of mice spectral sensitivity to UV and green lights, has been performed both by electrophysiology (Ekesten and Gouras, 2008) and $\mathrm{Ca}^{2+}$ imaging (Aihara et al., 2017; Tan et al., 2015). These studies illustrated that the majority of neurons responded to both and were similarly orientation-tuned also to both colors, especially the ones with higher orientation selectivity (Tan et al., 2015).

The similarity of neuronal V1 responses might be due to the fact that the 
A

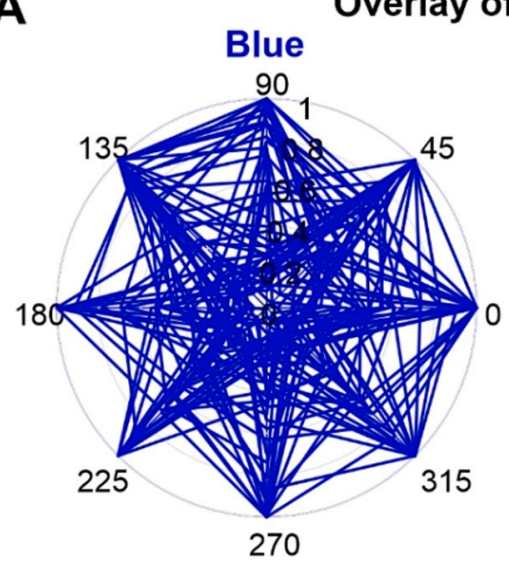

B

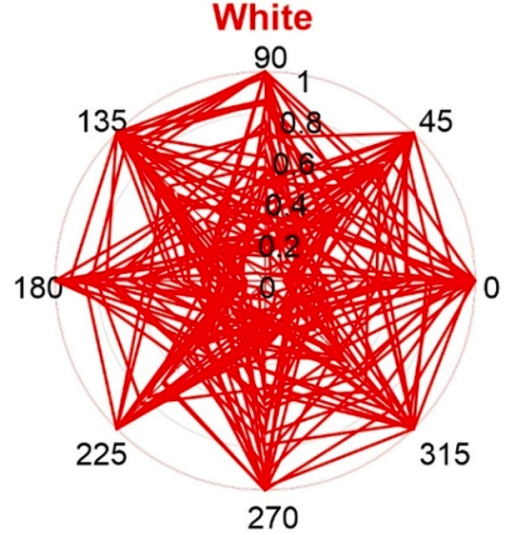

Average Polar plots

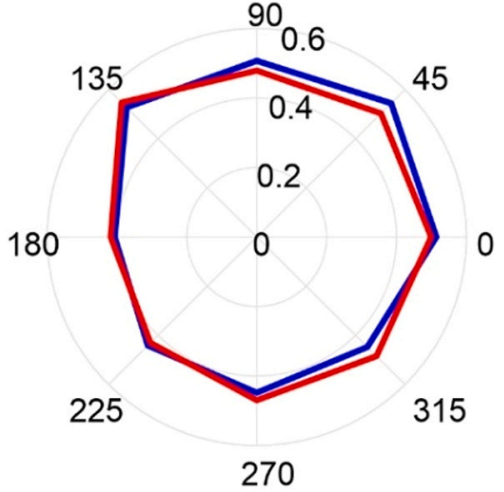

C

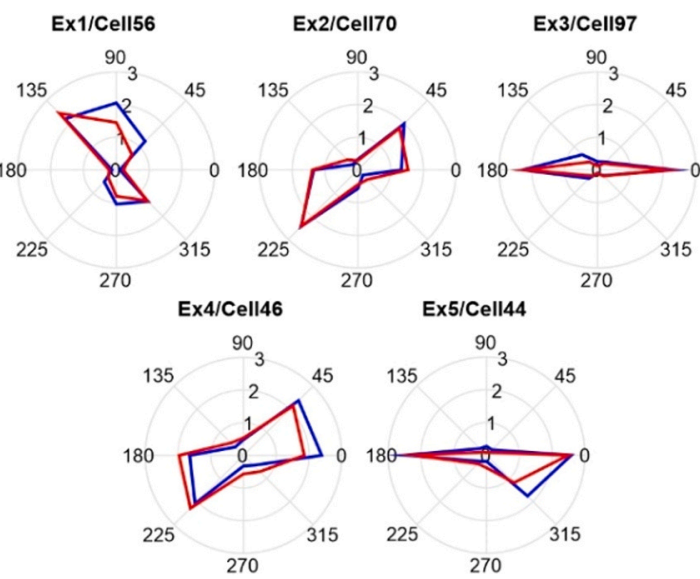

Ex3/Cell80

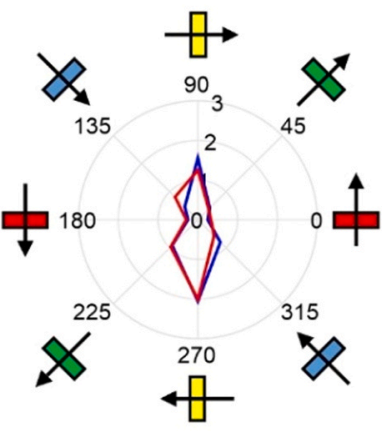

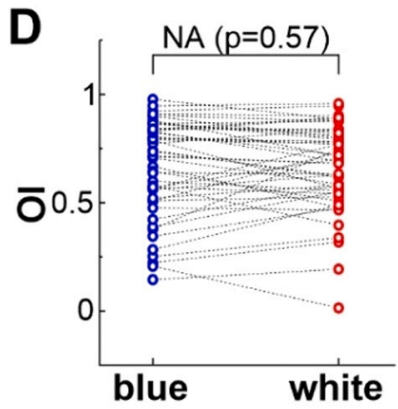
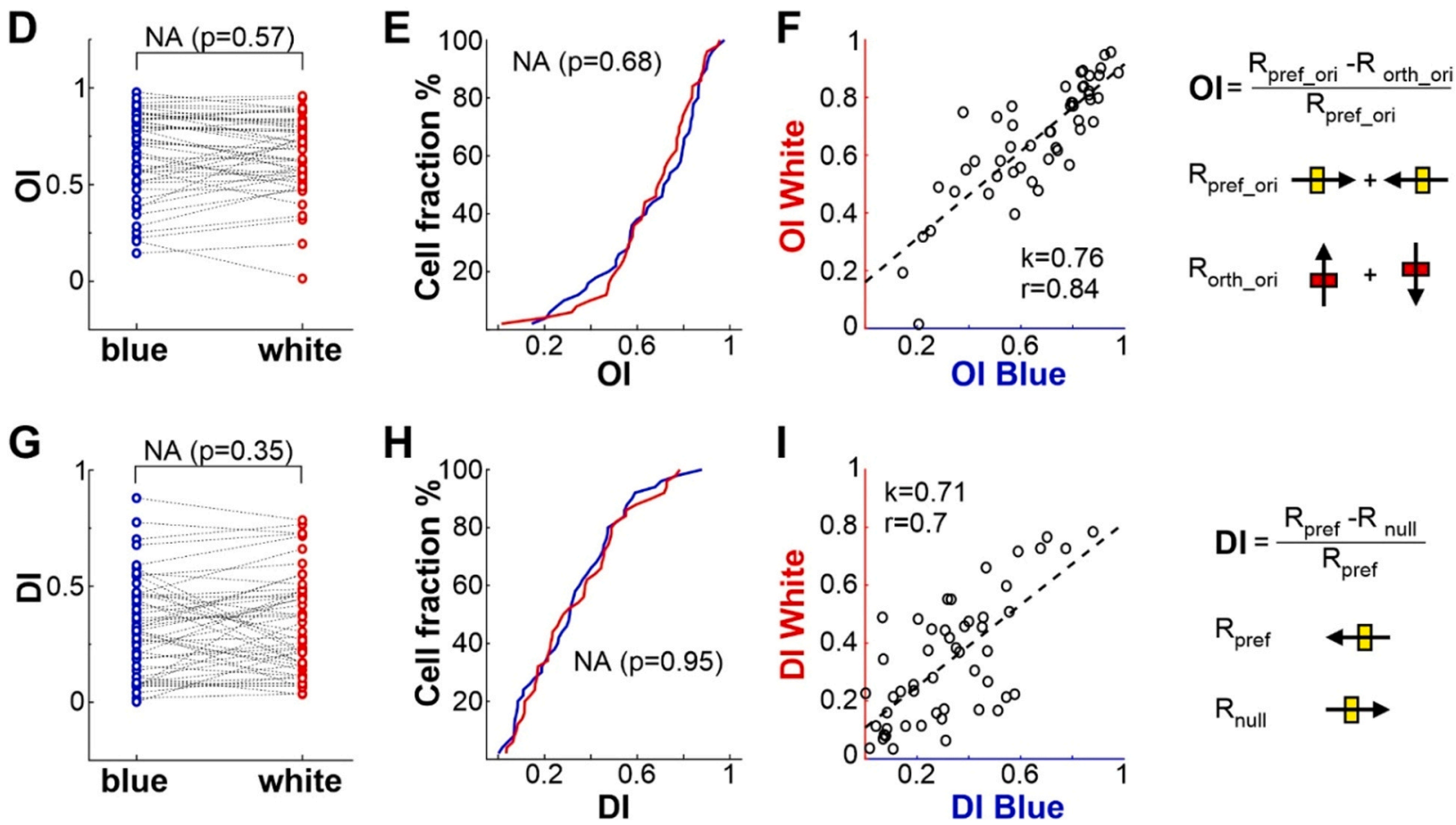

(caption on next page) 


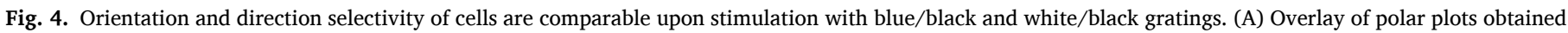

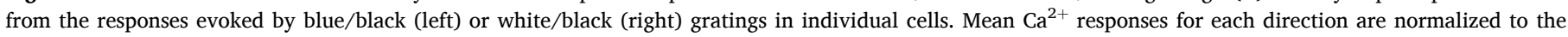

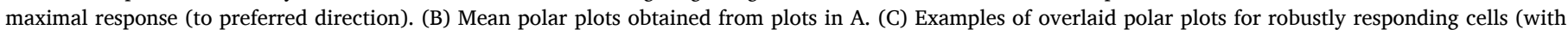

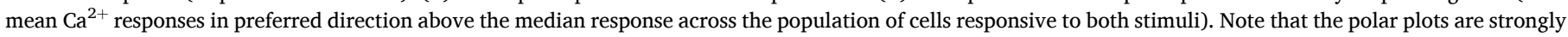

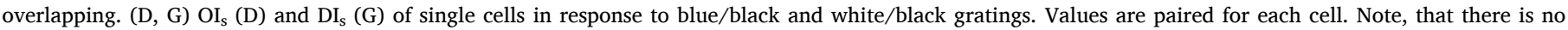

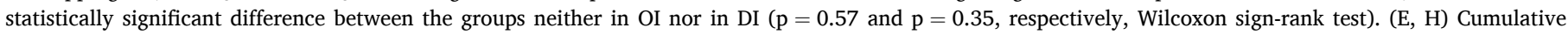

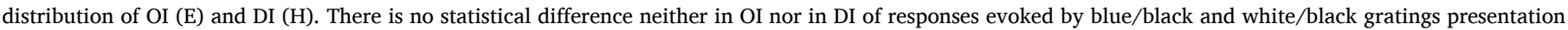

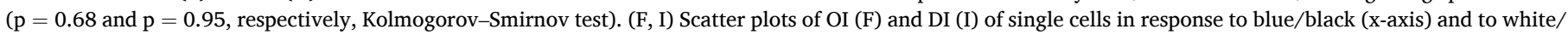

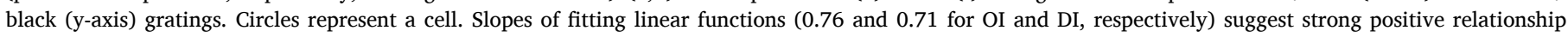

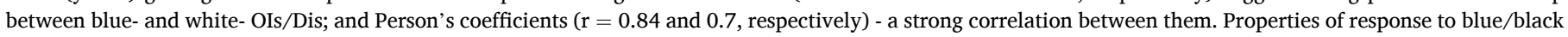
gratings are in blue, to white/black - in red. All plots are for neurons that respond to both gratings.
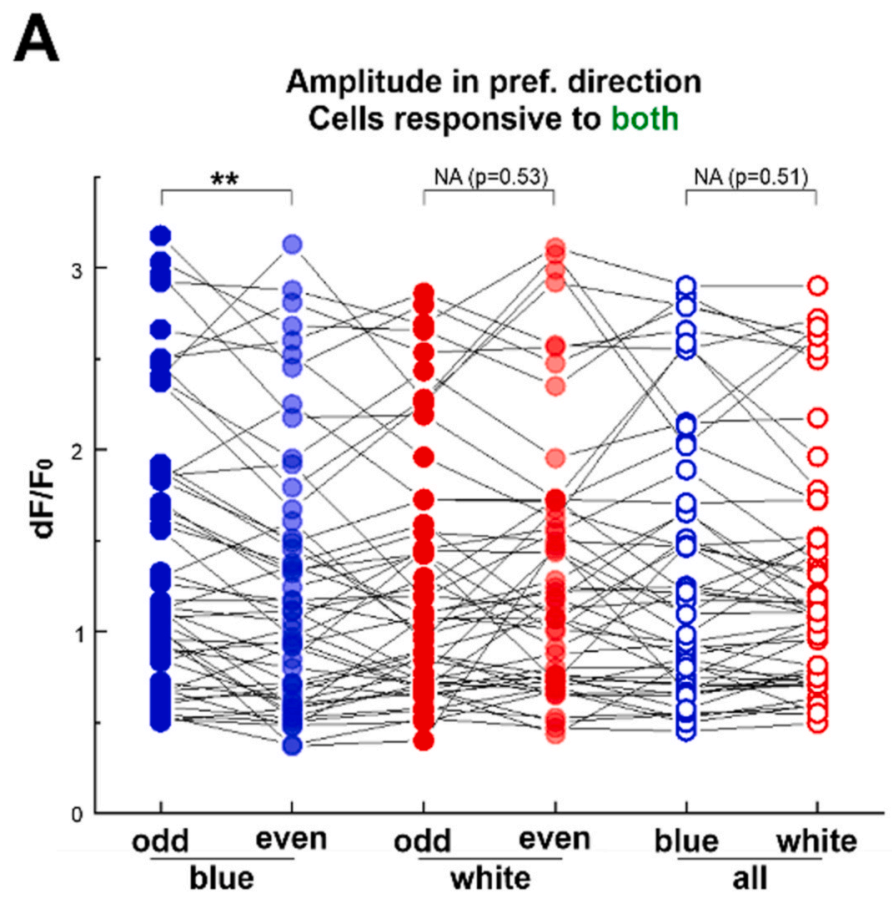

B

\section{Amplitude diff. in pref. direction Cells responsive to both}

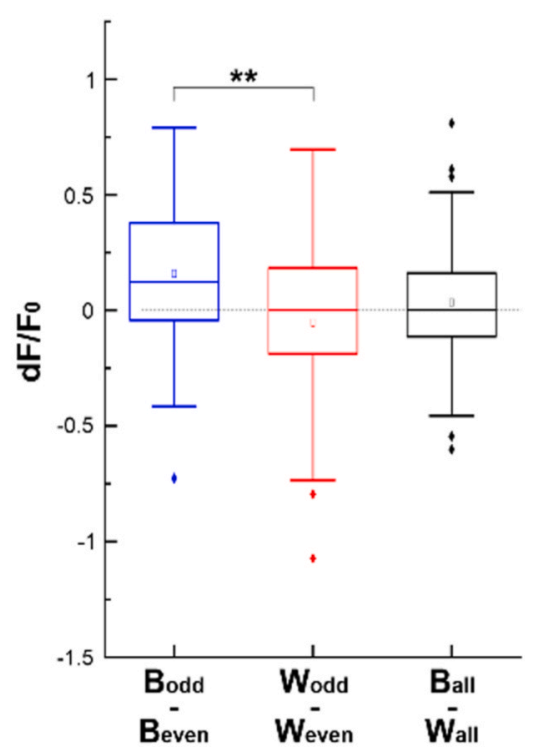

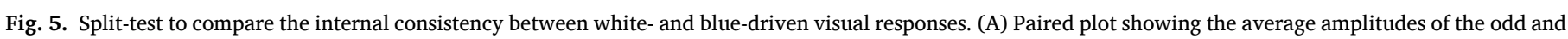

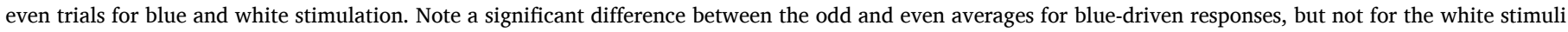

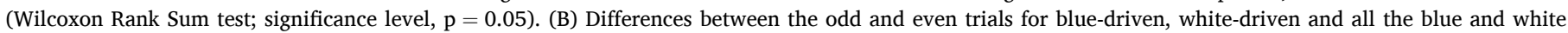

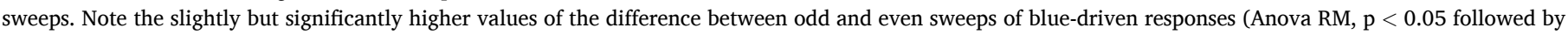
post hoc tests, significance level $\mathrm{p}=0.05$; Bodd-Beven vs Wodd-Weven, $\mathrm{p}=0.002$ ).

two stimuli activated the same retinal photoreceptors to a similar extent. Our convolution results are in line with the possibility that the overall similar degree of cortical responsiveness is indeed largely attributable to a similar level of integrated retinal recruitment.

Rods and cones are the main input of the image-forming visual pathway relaying information to V1 and are the limiting factor for visual perception. Indeed, knock-in mice expressing a human longwavelength-sensitive (L) cone photopigment, alongside the usual pigments, can discriminate green from red (Huberman and Niell, 2011; Jacobs et al., 2007), an ability that mice lack otherwise (Jacobs et al., 2004, 2007). Additionally, wild-type mice can distinguish UV and visible radiation, exploiting the separated chromatic sensitivities of cone UV and green opsins (Jacobs et al., 2004). The integrated retinal response reflects the summation of native photopigments' contribution (Rocha et al., 2016), and behavioral spectral sensitivity mirrors the retinal one (Jacobs et al., 1991, 2004, 2007). Finally, the chromatic tuning of V1 neurons depends on retinotopy and reflects the spatial distribution of retinal photopigments (Aihara et al., 2017; Ekesten and Gouras, 2008; Rhim et al., 2017). Here we did not systematically study whether the similarity in responses depends on specific retinotopic positions, as the screen placement was made in order to stimulate a wide portion of retina, including the regions closer to the vertical meridian, that corresponds to the entire binocular portion of V1 (see Material and Methods). However, even if the density of cones (expressing the S-opsin) displays retinotopic differences in rodents, we consider it plausible that our results would be similar also in more peripheral regions, where rods are dominant, in light of the close similarity between the S-opsins and rhodopsin's absorption spectra (see paragraph above).

What could have been the relative contribution of rods and cones? In other words, is the similar drive of V1 neurons observed for blue and white stimuli valid in scotopic or photopic conditions? We estimate that the visual responses we recorded were at least partly attributable to rods. Rods (97\% of photoreceptors; (Peirson et al., 2018)), are very light-sensitive, but able to adapt to light conditions traditionally considered to be photopic in rodents (Tikidji-Hamburyan et al., 2017). So at the light powers used ( $3 \mu \mathrm{W}$ at eye surface, corresponding to $\sim 10^{2}$ $\mathrm{cd} / \mathrm{m}^{2}$ or to $10^{10}$ photons $/ \mathrm{s} \mathrm{mm}^{2}$ ), rod photo-transduction should work at saturating levels (Naarendorp et al., 2010; Umino et al., 2008). Our convolutions shows that the similar responsivity we found might be attributable to the combined effect of the "green" opsinsispectral sensitivity functions (either of cones or of rods) and of the higher power of the blue stimulus precisely in the optical window.

Cones are very sparse ( $\sim 3 \%$ of photoreceptors; (Peirson et al., 2018)) and orders of magnitude less sensitive than rods (Naarendorp et al., 


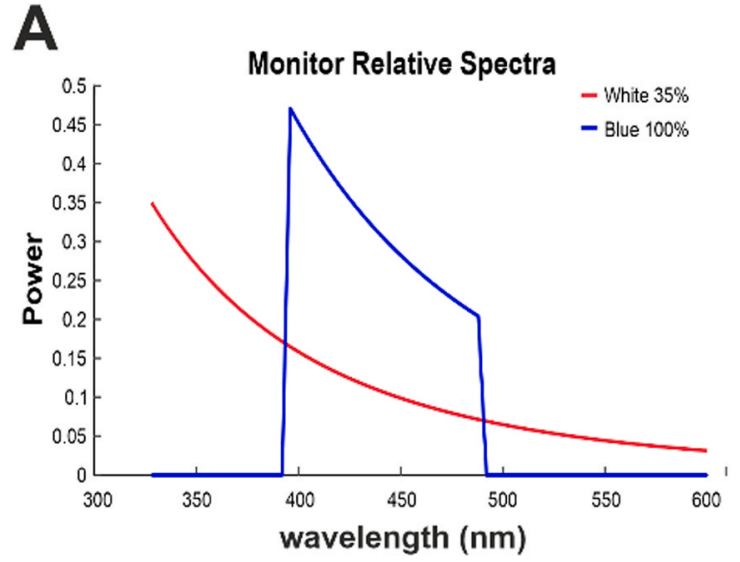

C

Total visual response to White and Blue

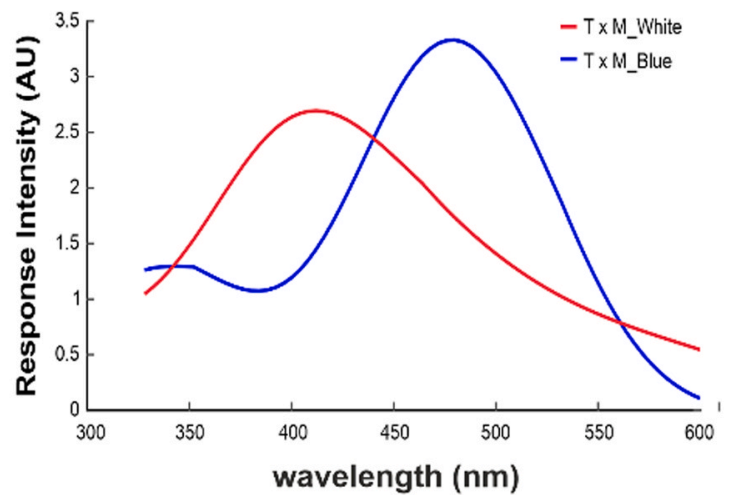

$\mathbf{B}$

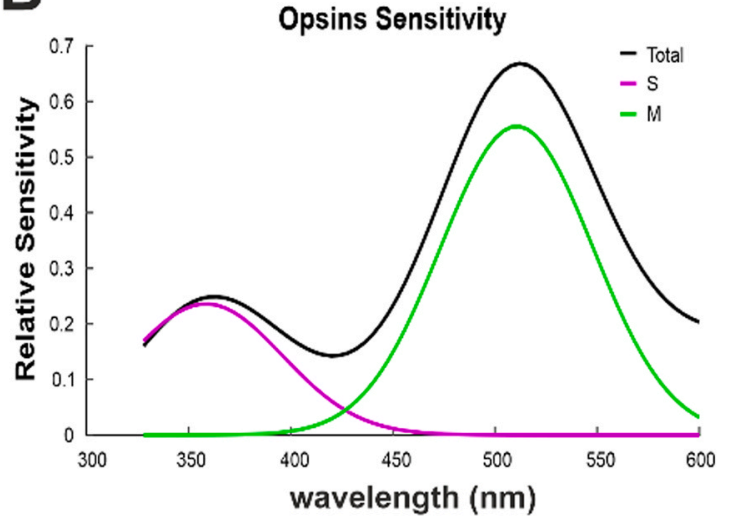

D

Visual response to White and Blue for M, S opsins

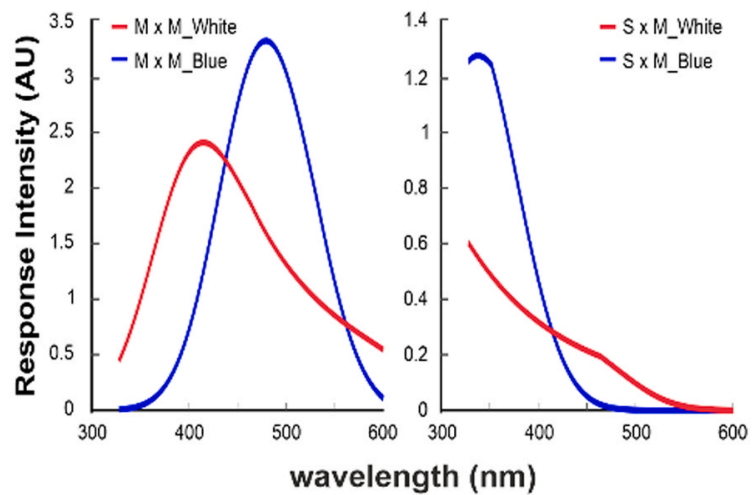

Fig. 6. Convolution between retinal spectrum sensitivity and the spectrum power profiles of the blue and white stimuli. (A) Energy density calculated by the CRT monitor for the white and blue visual stimuli (black and blue lines, respectively). (B) Spectral sensitivity functions of the opsin S (red), M (green) and the integrated retinal spectral sensitivity as measured by electroretinography (Rocha et al., 2016). (C) Convolution curves between the ERG spectral sensitivity curve and the energy density curves for blue and white, respectively. D. Similar convolutions calculated for the S and M opsins (right and left plots, respectively).

2010), but in mice they might take over vision at lower luminances than in humans (Naarendorp et al., 2010). Both blue and white visual stimuli used can activate cone's "green" sensitive M/L-opsin (expressed in 74\% of all cones; (Ortín-Martínez et al., 2014)), whose spectral sensitivity is very similar to that of rhodopsin. Since cones saturates above $\sim 0.1 \mathrm{~cd} / \mathrm{m}^{2}$ (Umino et al., 2008), we estimate that white and blue stimuli activated cones to a similar extent. Our convolutions show the predominant role of the green $(\mathrm{M})$ opsin in generating the retinal response to the equiluminant white and blue stimuli we used.

Although different, the blue and white spectra were "functionally" similar in their capability of recruiting the integrated retinal responses, because: (1) the red component (present in the white but not in the blue stimuli) can recruit the mouse retina only at much higher light intensities (Peirson et al., 2018); (2) mice do not possess cones with blue photopigment, so, a stronger blue $\lambda$ component in the blue light than that in white should not activate any cones better. Finally, photosensitive retinal ganglion cells (pRGC), which express melanopsin with blue peak sensitivity $(\sim 478 \mathrm{~nm})$, are not of our concern, since they are not implicated in image-forming, V1 responses (Peirson et al., 2018).

\subsection{Impact of similar V1 responsiveness to white and blue stimuli on behavior}

Can mice distinguish behaviorally white from blue? Mice can discriminate colors (Denman et al., 2018; Jacobs et al., 2004), but only in certain conditions (e.g. if stimuli are above horizon and if chromatic changes are large). The screen position in our case was covering the upper-central binocular visual field but also part of the monocular one. In these works mice were tested to discriminate colors with non-overlapping spectra (Jacobs et al., 2004), or change in UV-green (importantly UV and green had non-overlapping spectra, matching sensitivities of UV and green opsins (Denman et al., 2018)).

The color-discrimination ability is constrained by the spectral sensitivity differences of photopigments (cone's UV and green opsins, and rod's rhodopsin) and determined by chromatic-opponent cells responding at "ON" to one light spectra and at "OFF" to another. Surprisingly, not only cones, but also rods can be implicated in color opponency (Joesch and Meister, 2016). UV-green opponent cells were found in some RGC (12\% (Ekesten and Gouras, 2005; Ekesten et al., 2000)), and in V1 ( 1\% (Ekesten and Gouras, 2008; Tan et al., 2015)). We do not know whether white-blue opponent cells exist in mice V1. It is difficult to extrapolate this information from the above mentioned studies, since the latter compared non-overlapping colors. However, since white and blue spectra overlap and do not engage different types of photopigments, the existence of opponent cells is questionable.

Apart from chromatic opponency, which is related with the receptive field spatial substructure, chromatic-selective neurons could contribute to color-discrimination ability. Color-selective cells in L2/3 of mice V1 were also shown before (Aihara et al., 2017; Ekesten and Gouras, 2008; Tan et al., 2015). Using our criteria for responsiveness (see 2.3.5.), we found neurons activated only by white or by blue. However, as we described in Section 3.2., their responses were on average smaller than in cells responding to both colors and in some cells rather sparse. Thus, scarcer responsiveness might have caused a spurious classification as 
"unichromatic" in some of these cells. Strict behavioral comparisons of chromatic discrimination capacity between two overlapping spectra are missing, but given the existence of chromatic selectivity in mouse V1, this possibility is plausible, taking into account some degree of separation between the two cell populations we found in our reporter line.

Up to which degree can these results be generalized to other mice strains? Our experiments were performed in pigmented mice. Albino mice have relatively fewer total cones (Ortín-Martínez et al., 2014; Whitney et al., 2011), fewer cones expressing green opsin and more UV-opsin-expressing cones with less-pronounced dorso-ventral gradient of the last (Ortín-Martínez et al., 2014), and fewer rods (Donatien and Jeffery, 2002). However, the retinal selectivity of the pigments is similar, thus suggesting that our findings could be extended to albino strains.

Our stimuli do not significantly recruit the UV sensitivity of the mouse retina. Adding UV radiation to blue light would engage UV mice spectral sensitivity, without causing light contamination during $\mathrm{Ca}^{2+}$ imaging. CRT monitors lack UV-spectra, but recent stimulators can do it (Franke et al., 2019).

A limitation of our study is that we compared visual responses to blue and white stimuli in saturating conditions, that is with gratings of optimal spatial frequencies, temporal frequencies and contrasts. Spatial and temporal frequency tuning curves, as well as a contrast sensitivity curve should be tested in order to have a more robust physiological basis for an educated guess on the behavioral equivalence of the two types of chromatic stimuli presented at the perceptual thresholds.

\subsection{Generalized validity of results in different illumination conditions}

We have documented a similar responsiveness to white and blue stimuli with electrophysiology in wild type mice and with population calcium imaging in a GCaMP6s mouse. The selected strain (4.3) has similarly high expression levels in layer 5 and layer $2 / 3$ in visual cortex and it is characterized by a relatively low number of responsive neurons (ca 10\%) but displaying brisk responses (see Figs. 3 and 4 in Dana et al. (2014)). The strain has been used for several relevant work in cortical integrative physiology in various areas in the premotor (Chen et al., 2017), visual cortices (Jenks and Shepherd, 2020; Lee et al., 2017) as well as on a large field of view on multiple cortical areas (Sofroniew et al., 2016). However, a word of caution on generalizing the validity of the calcium imaging result should be expressed because the percentage of responsive neurons in our strain is low (although responses are brisk) and significantly lower compared to other Thy1 lines (such as 4.12) or AAV vectors (see (Dana et al., 2014), Fig. 4h; also e.g. (de Vries et al., 2020)). With respect to this concern, the similarity of VEP responses to white and blue light in wild types mice that we reported suggests that there is a significant chance that our results are independent of the technical approach or labeling technique used.

Both rods and cones should have contributed to the visual responses we recorded. We performed experiments in conditions commonly used for in vivo electrophysiology and 2P microscopy: low level of ambient light and moderate intensity of visual stimuli (here $3 \mu \mathrm{W}=0.95510^{-2} \mathrm{~W} / \mathrm{m}^{2}$ or $\sim 10^{2} \mathrm{~cd} / \mathrm{m}^{2}$, or $\sim 10^{10}$ photons $/ \mathrm{s} \mathrm{mm}^{2}$, or $<1 \mathrm{~lx}$ ) at eye level. This corresponds to mice photopic range (Umino et al., 2008). However, in these conditions rods continue to respond $\left(\sim 310^{4} \mathrm{R} * / \operatorname{rod} \mathrm{s}\right.$ (photoisomerization per rod) (Naarendorp et al., 2010) have been measured for luminances comparable to our conditions $10^{2}-10^{3} \mathrm{~cd} / \mathrm{m}^{2}$ (Umino et al., 2008)), even though they might temporarily loose sensitivity after abrupt luminance changes. In steady illumination, as in our experiments, rod's contrast sensitivity, initially reduced in photopic range, was shown to recover (Tikidji-Hamburyan et al., 2017).

We expect similar responsiveness in photopic and high mesopic (above $\sim 0.1 \mathrm{~cd} / \mathrm{m}^{2}$ (Umino et al., 2008)) conditions. Indeed, both cones (Umino et al., 2008) and rods (Naarendorp et al., 2010) should respond at saturating levels in the luminance conditions used. That our illumination level approaches saturation is suggested by the fact that a 3-fold light intensity increase (from our white $35 \%$ to white $100 \%$ intensity) caused on average only $\sim 53 \%$ (much lower than $300 \%$ ) increase in VEP amplitude $(-0.15 \pm 0.05$ vs $-0.23 \pm 0.09 \mathrm{mV}$ for $35 \%$ and $100 \%$, respectively - see Fig. 7). This contradicts older reports, showing quasi-linear amplitude increases upon luminance increment (Porciatti et al., 1999). This finding is in line with recent behavioral studies. In one in mesopic conditions, the authors documented that mice ability to discriminate two subsequent stimuli reached plateau already when the luminance difference between stimuli was just above 30\% (Denman et al., 2018). Also, mice contrast sensitivity plateaued at $\sim 10^{-2} \mathrm{~cd} / \mathrm{m}^{2}$ (Umino et al., 2008) - a luminance corresponding to our $35 \%$ white stimulus.

We might expect possible differences in white-driven and blue-driven responses at low-scotopic conditions. On one side, the convolution estimates for the $\mathrm{M}$ opsin (having a spectrum very similar to rhodopsin) suggest a similar integrated retinal recruitment for equiluminant stimuli. However, the spectrum influences pupil constriction (Lucas et al., 2001), and differences in pupil size upon blue and white stimuli can be noticeable in low scotopic conditions, but small in our luminance range (with pupil constriction $\sim 90 \%$ ).

\subsection{Advantages of blue visual stimulation combined with different imaging techniques/approaches}

Blue visual stimulation, beyond allowing to omit time-consuming shielding, can facilitate to combine $\mathrm{Ca}^{2+}$ imaging with optogenetics - so-called alloptical approach (Carrillo-Reid et al., 2017; Forli et al., 2018; Yang et al., 2018), or with electrophysiological recordings - e.g. (Chen et al., 2013). Electrophysiology requires indeed free access for recording electrodes, also rendering shielding problematic when combined with 2P experiments. All-optical microcircuit interrogation becomes even more problematic in head-fixed behavioral setups requiring rather "open" spatial configurations.

In relation to white, blue light stimulation can be beneficial while imaging not only GCaMP6s or other "green" cpEGFP-based sensors $(\lambda \mathrm{em}=515 \mathrm{~nm})$ GECI (Chen et al., 2012, 2013; Dana et al., 2014, 2019), but also red-shifted GECI (as - jRGECO1a, jRCaMP1a and b; $\lambda$ em $600 \mathrm{~nm}$, (Dana et al., 2016; Dana et al., 2018)), since white $(380-730 \mathrm{~nm})$ visual stimuli would contaminate the acquired emission of the red-shifted dyes as well. The application can be extended also to other sensors used to image neuronal function such as synaptic activity reporters, e.g. SynaptopHluorin (an indicator of synaptic release), or SuperGluSnFR and iGluSnFR (glutamate sensors) or voltage-sensitive dyes (Broussard et al., 2014; Carandini et al., 2015; Chemla and Chavane, 2010).

Despite utility of blue light, long-lasting high intensity blue visual stimulation should be avoided, as UV-violet-blue components of fluorescent lamps (with $4.9710^{-05} \mathrm{~mW} / \mathrm{cm}^{2} / \mathrm{s}$ radiant exposure) cause lightinduced retinal damage within $1 \mathrm{~h}$ of exposure (Narimatsu et al., 2014).

\subsection{Comparison with alternative approaches}

In addition to shielding, light contamination can be minimized by temporally separating the recording of fluorescence of $\mathrm{Ca}^{2+}$ indicator and visual stimulus presentation (Leinweber et al., 2014; Reiff et al., 2010), exploiting also the relative sluggishness of calcium responses compared to the voltage response. Stimuli are shown during the time periods when the data is not acquired/eliminated (during the turnaround points in case of raster scanning in the $2 \mathrm{P}$ scope). This, however, implies precise synchronisation of the screen's/projector's light output to the scanning/data collection of the imaging system and requires tailor-made electronics to precisely synchronize stimulation-acquisition times. In addition, this approach can impose constraints in the temporal structure of the presented stimuli that for example would be problematic with certain scanning modalities, such as the random scan (AOD-based) approach used here. 
A

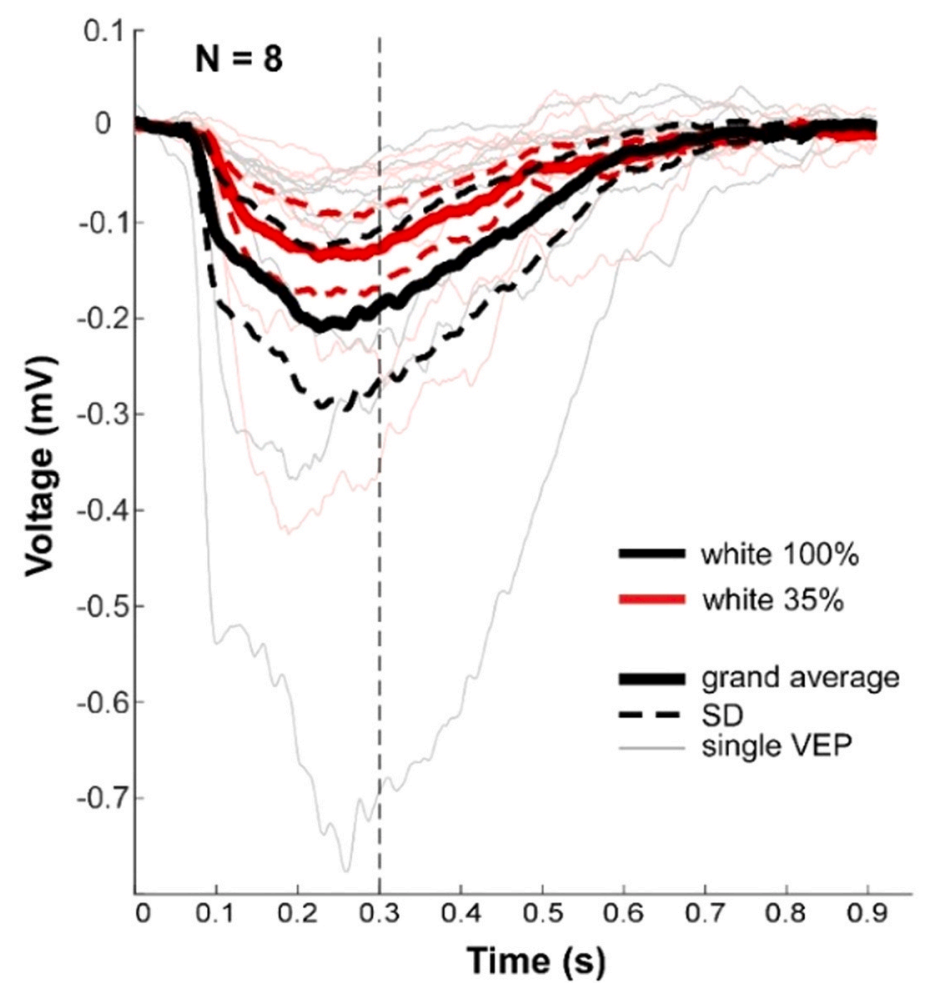

B

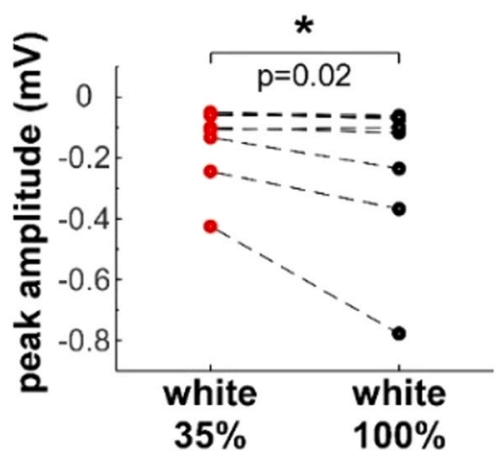

C

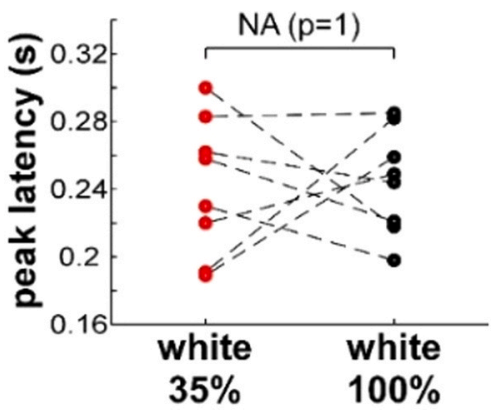

Fig. 7. VEP in response to white/black gratings with $100 \%$ and $35 \%$ intensity of white in mice V1. (A) Grand averages $(\mathrm{N}=8$ mice, thick lines) $\pm \mathrm{SD}$ (thinner dashed lines) and individual VEPs (thin lines) in response to 100\% (in black) and 35\% (in read) intensity of white. Dashed vertical line confines the time for peak search (0.3 s). Note that the responses are strongly overlapping. (B, C) Amplitude (B) and latency (C) of single VEP's peaks in response to 100\% (in black) and 35\% (in read) white intensity. Note, that VEP amplitude in response to $100 \%$ intensity is significantly (Wilcoxon signed rank test, $\mathrm{p}=0.02, \mathrm{~N}=8$ ), but not 3 times higher than that in response to $35 \%$ intensity.

\section{Conclusions}

Blue light, spectrally selected so not to interfere with calcium imaging with green-shifted GCaMP6s' emissions, activates mouse V1 to a similar extent as an equi-powerful white light. This was assessed by measurements of both integrated synaptic responses (VEPs, reflecting the input) and $\mathrm{Ca}^{2+}$ responses of single neurons in the network (mirroring their action potential output (Kerr et al., 2005)). White and blue stimuli recruit the same network of neurons to a similar extent, without differences in fundamental functional response properties such as orientation and direction selectivities.

\section{Supplementary data}

By visual examination we found 3 clear "off-cells", activated solely by the termination of the grating stimulation (with activation onset after stimulus OFF). Interestingly, the offset of both blue and white grating stimulation could trigger the increase in GCaMP6s fluorescence in these cells (see Fig. S2.2). As the time windows to calculate the response and blank values in our analysis were adapted for "on-cells", "off-cells" were not determined as responsive and not included in further analysis.

\section{CRediT authorship contribution statements}

Paolo Medini supervised the project; Paolo Medini and Tatiana Kuznetsova did experimental planning; Tatiana Kuznetsova performed the experiments and wrote the manuscript; Paolo Medini, Kamil Antos, Evgenya Malinina, Stylianos Papaioannou - revised the manuscript, Kamil Antos - developed the Matlab code for VEP and $\mathrm{Ca}^{2+}$ imaging analysis, analyzed $\mathrm{Ca}^{2+}$ imaging in Matlab, developed visual simulation software and software communication for LFP, IOI and AODexperiments; Tatiana Kuznetsova analyzed the data in Origin; Kamil Antos and Tatiana Kuznetsova prepared the graphs; Tatiana Kuznetsova prepared final illustrations; Evgenya Malinina and Kamil Antos developed stimulation protocol for AOD and IOI; Evgenya Malinina optimized the surgery and experimental design for $\mathrm{Ca}^{2+}$ imaging and IOI and preliminary experiments; Stylianos Papaioannou contributed to $\mathrm{Ca}^{2+}$ imaging analysis and computed the convolutions.

\section{Acknowledgments}

Supported by grant Knut och Alice Wallenberg KAW 2014.0051 and Swedish Research Council (Vetenskapsrådet) VR 2014.02350. We thank Sebastian Sulis Sato for the help with mastering the AOD set up, experimental and critical suggestions and Thy-1-GCaMP6s colony management; Sebastian Sulis Sato and Anushree Tripathi for showing the IOI; Johan Zakrisson and Sebastian Sulis Sato for tuning Ti-Saphire laser in AOD set up; Johan Zakrisson for cone slit design and light power measurements; Johan Zakrisson and Luciano Censoni for support in photometry units transfers; Per Utsi for help with mechanical engineering.

\section{Conflict of interest}

None.

\section{Appendix A. Supporting information}

Supplementary data associated with this article can be found in the online version at doi:10.1016/j.jneumeth.2021.109287. 


\section{References}

Aihara, S., Yoshida, T., Hashimoto, T., Ohki, K., 2017. Color representation is retinotopically biased but locally intermingled in mouse V1. Front. Neural Circuits 11 (22), 22.

Ali, F., Kwan, A.C., 2020. Interpreting in vivo calcium signals from neuronal cell bodies, axons, and dendrites: a review. Neurophotonics 7, 011402.

Broussard, G.J., Liang, R., Tian, L., 2014. Monitoring activity in neural circuits with genetically encoded indicators. Front. Mol. Neurosci. 7, 97.

Carandini, M., Shimaoka, D., Rossi, L.F., Sato, T.K., Benucci, A., Knöpfel, T., 2015. Imaging the awake visual cortex with a genetically encoded voltage indicator. J. Neurosci. 35, 53-63.

Carrillo-Reid, L., Yang, W., Kang Miller, J.E., Peterka, D.S., Yuste, R., 2017. Imaging and optically manipulating neuronal ensembles. Annu Rev. Biophys. 46, 271-293.

Chemla, S., Chavane, F., 2010. Voltage-sensitive dye imaging: technique review and models. J. Physiol. Paris 104, 40-50.

Chen, Q., Cichon, J., Wang, W., Qiu, L., Lee, S.J., Campbell, N.R., Destefino, N., Goard, M.J., Fu, Z., Yasuda, R., Looger, L.L., Arenkiel, B.R., Gan, W.B., Feng, G., 2012. Imaging neural activity using Thy1-GCaMP transgenic mice. Neuron 76, 297-308.

Chen, T.-W., Wardill, T.J., Sun, Y., Pulver, S.R., Renninger, S.L., Baohan, A., Schreiter, E. R., Kerr, R.A., Orger, M.B., Jayaraman, V., Looger, L.L., Svoboda, K., Kim, D.S., 2013. Ultrasensitive fluorescent proteins for imaging neuronal activity. Nature 499, 295-300.

Chen, T.W., Li, N., Daie, K., Svoboda, K., 2017. A map of anticipatory activity in mouse motor cortex. Neuron 94, 866-879 e4.

Dana, H., Chen, T.-W., Hu, A., Shields, B.C., Guo, C., Looger, L.L., Kim, D.S., Svoboda, K., 2014. Thy1-GCaMP6 transgenic mice for neuronal population imaging in vivo. PLOS One 9, 108697.

Dana, H., Mohar, B., Sun, Y., Narayan, S., Gordus, A., Hasseman, J.P., Tsegaye, G., Holt, G.T., Hu, A., Walpita, D., Patel, R., Macklin, J.J., Bargmann, C.I., Ahrens, M.B., Schreiter, E.R., Jayaraman, V., Looger, L.L., Svoboda, K., Kim, D.S., 2016. Sensitive red protein calcium indicators for imaging neural activity. eLife 5, e12727.

Dana, H., Novak, O., Guardado-Montesino, M., Fransen, J.W., Hu, A., Borghuis, B.G., Guo, C., Kim, D.S., Svoboda, K., 2018. Thy1 transgenic mice expressing the red fluorescent calcium indicator jRGECO1a for neuronal population imaging in vivo. PLOS One 13, 0205444.

Dana, H., Sun, Y., Mohar, B., Hulse, B.K., Kerlin, A.M., Hasseman, J.P., Tsegaye, G., Tsang, A., Wong, A., Patel, R., Macklin, J.J., Chen, Y., Konnerth, A., Jayaraman, V., Looger, L.L., Schreiter, E.R., Svoboda, K., Kim, D.S., 2019. High-performance calcium sensors for imaging activity in neuronal populations and microcompartments. Nat. Methods 16, 649-657.

de Vries, S.E.J., Lecoq, J.A., Buice, M.A., Groblewski, P.A., Ocker, G.K., Oliver, M., Feng, D., Cain, N., Ledochowitsch, P., Millman, D., Roll, K., Garrett, M., Keenan, T., Kuan, L., Mihalas, S., Olsen, S., Thompson, C., Wakeman, W., Waters, J., Williams, D., Barber, C., Berbesque, N., Blanchard, B., Bowles, N., Caldejon, S.D., Casal, L., Cho, A., Cross, S., Dang, C., Dolbeare, T., Edwards, M., Galbraith, J., Gaudreault, N., Gilbert, T.L., Griffin, F., Hargrave, P., Howard, R., Huang, L., Jewell, S., Keller, N., Knoblich, U., Larkin, J.D., Larsen, R., Lau, C., Lee, E., Lee, F. Leon, A., Li, L., Long, F., Luviano, J., Mace, K., Nguyen, T., Perkins, J., Robertson, M., Seid, S., Shea-Brown, E., Shi, J., Sjoquist, N., Slaughterbeck, C., Sullivan, D., Valenza, R., White, C., Williford, A., Witten, D.M., Zhuang, J., Zeng, H., Farrell, C., Ng, L., Bernard, A., Phillips, J.W., Reid, R.C., Koch, C., 2020. A large-scale standardized physiological survey reveals functional organization of the mouse visual cortex. Nat. Neurosci. 23, 138-151.

Denman, D.J., Luviano, J.A., Ollerenshaw, D.R., Cross, S., Williams, D., Buice, M.A., Olsen, S.R., Reid, R.C., 2018. Mouse color and wavelength-specific luminance contrast sensitivity are non-uniform across visual space. eLife 7, e31209.

Donatien, P., Jeffery, G., 2002. Correlation between rod photoreceptor numbers and levels of ocular pigmentation. Invest Ophthalmol. Vis. Sci. 43, 1198-1203.

Ekesten, B., Gouras, P., 2005. Cone and rod inputs to murine retinal ganglion cells: evidence of cone opsin specific channels. Vis. Neurosci. 22, 893-903.

Ekesten, B., Gouras, P., 2008. Cone inputs to murine striate cortex. BMC Neurosci. 9, 113.

Ekesten, B., Gouras, P., Yamamoto, S., 2000. Cone inputs to murine retinal ganglion cells. Vis. Res. 40, 2573-2577.

Forli, A., Vecchia, D., Binini, N., Succol, F., Bovetti, S., Moretti, C., Nespoli, F., Mahn, M., Baker, C.A., Bolton, M.M., Yizhar, O., Fellin, T., 2018. Two-photon bidirectional control and imaging of neuronal excitability with high spatial resolution in vivo. Cell Rep. 22, 3087-3098.

Franke, K., Maia Chagas, A., Zhao, Z., Zimmermann, M.J.Y., Bartel, P., Qiu, Y., Szatko, K. P., Baden, T., Euler, T., 2019. An arbitrary-spectrum spatial visual stimulator for vision research. eLife 8, e48779.

Galvan, A., Stauffer, W.R., Acker, L., El-Shamayleh, Y., Inoue, K.I., Ohayon, S., Schmid, M.C., 2017. Nonhuman primate optogenetics: recent advances and future directions. J. Neurosci. 37, 10894-10903.

Goard, M.J., Pho, G.N., Woodson, J., Sur, M., 2016. Distinct roles of visual, parietal, and frontal motor cortices in memory-guided sensorimotor decisions. eLife 5, e13764.

Goltstein, P.M., Montijn, J.S., Pennartz, C.M., 2015. Effects of isoflurane anesthesia on ensemble patterns of Ca2 + activity in mouse v1: reduced direction selectivity independent of increased correlations in cellular activity. PLoS One 10, 0118277.

Grienberger, C., Konnerth, A., 2012. Imaging calcium in neurons. Neuron 73, 862-885.

Grinvald, A., Shoham, D., Shmuel, A., Glaser, D., Vanzetta, I., Shtoyerman, E., Slovin, H., Wijnbergen, C., Hildesheim, R., Arieli, A., 1999. In-vivo optical imaging of cortical architecture and dynamics. In: Windhorst, U., Johansson, H. (Eds.), Modern Techniques in Neuroscience Research. Springer Berlin Heidelberg, Berlin, Heidelberg, pp. 893-969.
Huberman, A.D., Niell, C.M., 2011. What can mice tell us about how vision works? Trends Neurosci. 34, 464-473.

Hwang, E.J., Link, T.D., Hu, Y.Y., Lu, S., Wang, E.H.-J., Lilascharoen, V., Aronson, S., O'Neil, K., Lim, B.K., Komiyama, T., 2019. Corticostriatal flow of action selection bias. Neuron 104, 1126-1140 e6.

Hübener, M., 2003. Mouse visual cortex. Curr. Opin. Neurobiol. 13, 413-420.

Itokazu, T., Hasegawa, M., Kimura, R., Osaki, H., Albrecht, U.-R., Sohya, K., Chakrabarti, S., Itoh, H., Ito, T., Sato, T.K., Sato, T.R., 2018. Streamlined sensory motor communication through cortical reciprocal connectivity in a visually guided eye movement task. Nat. Commun. 9, 338.

Jacobs, G.H., Neitz, J., Deegan 2nd, J.F., 1991. Retinal receptors in rodents maximally sensitive to ultraviolet light. Nature 353, 655-656.

Jacobs, G.H., Williams, G.A., Cahill, H., Nathans, J., 2007. Emergence of novel color vision in mice engineered to express a human cone photopigment. Science 315, 1723-1725.

Jacobs, G.H., Williams, G.A., Fenwick, J.A., 2004. Influence of cone pigment coexpression on spectral sensitivity and color vision in the mouse. Vis. Res 44, 1615-1622.

Jenks, K.R., Shepherd, J.D., 2020. Experience-dependent development and maintenance of binocular neurons in the mouse visual cortex. Cell Rep. 30, 1982-1994 e4.

Jiao, Z.F., Shang, C.F., Wang, Y.F., Yang, Z., Yang, C., Li, F.N., Xie, J.Z., Pan, J.W., Fu, L., Du, J.L., 2018. All-optical imaging and manipulation of whole-brain neuronal activities in behaving larval zebrafish. Biomed. Opt. Express 9, 6154-6169.

Joesch, M., Meister, M., 2016. A neuronal circuit for colour vision based on rod-cone opponency. Nature 532, 236-239.

Juavinett, A.L., Nauhaus, I., Garrett, M.E., Zhuang, J., Callaway, E.M., 2017. Automated identification of mouse visual areas with intrinsic signal imaging. Nat. Protoc. 12 $32-43$.

Kerlin, A.M., Andermann, M.L., Berezovskii, V.K., Reid, R.C., 2010. Broadly tuned response properties of diverse inhibitory neuron subtypes in mouse visual cortex. Neuron 67, 858-871.

Kerr, J.N.D., Greenberg, D., Helmchen, F., 2005. Imaging input and output of neocortical networks in vivo. Proc. Natl. Acad. Sci. U. S. A. 102, 14063-14068.

Krumin, M., Lee, J.J., Harris, K.D., Carandini, M., 2018. Decision and navigation in mouse parietal cortex. eLife 7, e42583.

Lecoq, J., Savall, J., Vucinic, D., Grewe, B.F., Kim, H., Li, J.Z., Kitch, L.J., Schnitzer, M.J., 2014. Visualizing mammalian brain area interactions by dual-axis two-photon calcium imaging. Nat. Neurosci. 17, 1825-1829.

Lee, S., Meyer, J.F., Park, J., Smirnakis, S.M., 2017. Visually driven neuropil activity and information encoding in mouse primary visual cortex. Front Neural Circuits 11, 50.

Leinweber, M., Ward, D.R., Sobczak, J.M., Attinger, A., Keller, G.B., 2017. A sensorimotor circuit in mouse cortex for visual flow predictions. Neuron 95, 1420-1432 e5.

Leinweber, M., Zmarz, P., Buchmann, P., Argast, P., Hübener, M., Bonhoeffer, T., Keller, G.B., 2014. Two-photon calcium imaging in mice navigating a virtual reality environment. J. Vis. Exp.: JoVE 50885.

Lucas, R.J., Douglas, R.H., Foster, R.G., 2001. Characterization of an ocular photopigment capable of driving pupillary constriction in mice. Nat. Neurosci. 4, 621-626.

Mazurek, M., Kager, M., Van Hooser, S.D., 2014. Robust quantification of orientation selectivity and direction selectivity. Front. Neural Circuits 8, 92.

Mazzaro, N., Barini, E., Spillantini, M.G., Goedert, M., Medini, P., Gasparini, L., 2016. Tau-driven neuronal and neurotrophic dysfunction in a mouse model of early tauopathy. J. Neurosci. 36, 2086-2100.

Medini, P., 2011. Cell-type-specific sub- and suprathreshold receptive fields of layer 4 and layer $2 / 3$ pyramids in rat primary visual cortex. Neuroscience 190, 112-126.

Naarendorp, F., Esdaille, T.M., Banden, S.M., Andrews-Labenski, J., Gross, O.P., Pugh Jr., E.N., 2010. Dark light, rod saturation, and the absolute and incremental sensitivity of mouse cone vision. J. Neurosci. 30, 12495-12507.

Narimatsu, T., Ozawa, Y., Miyake, S., Kubota, S., Yuki, K., Nagai, N., Tsubota, K., 2014. Biological effects of blocking blue and other visible light on the mouse retina. Clin. Exp. Ophthalmol. 42, 555-563.

Nauhaus, I., Nielsen, K.J., Callaway, E.M., 2012. Nonlinearity of two-photon Ca2+ imaging yields distorted measurements of tuning for V1 neuronal populations. J. Neurophysiol. 107, 923-936.

Niell, C.M., Stryker, M.P., 2008. Highly selective receptive fields in mouse visual cortex. J. Neurosci. 28, 7520-7536.

Ortín-Martínez, A., Nadal-Nicolás, F.M., Jiménez-López, M., Alburquerque-Béjar, J.J., Nieto-López, L., García-Ayuso, D., Villegas-Pérez, M.P., Vidal-Sanz, M., AgudoBarriuso, M., 2014. Number and distribution of mouse retinal cone photoreceptors: differences between an Albino (Swiss) and a Pigmented (C57/BL6) strain. PLOS One 9, 102392.

Peirson, S.N., Brown, L.A., Pothecary, C.A., Benson, L.A., Fisk, A.S., 2018. Light and the laboratory mouse. J. Neurosci. Methods 300, 26-36.

Porciatti, V., Pizzorusso, T., Maffei, L., 1999. The visual physiology of the wild type mouse determined with pattern VEPs. Vis. Res. 39, 3071-3081.

Reiff, D.F., Plett, J., Mank, M., Griesbeck, O., Borst, A., 2010. Visualizing retinotopic halfwave rectified input to the motion detection circuitry of Drosophila. Nat. Neurosci. 13, 973-978.

Rhim, I., Coello-Reyes, G., Ko, H.K., Nauhaus, I., 2017. Maps of cone opsin input to mouse V1 and higher visual areas. J. Neurophysiol. 117, 1674-1682.

Rocha, F.A., Gomes, B.D., Silveira, L.C., Martins, S.L., Aguiar, R.G., de Souza, J.M., Ventura, D.F., 2016. Spectral sensitivity measured with electroretinogram using a constant response method. PLoS One 11, 0147318. 
Shipley, F.B., Clark, C.M., Alkema, M.J., Leifer, A.M., 2014. Simultaneous optogenetic manipulation and calcium imaging in freely moving C. elegans. Front Neural Circuits $8,28$.

Simpson, J.H., Looger, L.L., 2018. Functional imaging and optogenetics in drosophila. Genetics 208, 1291-1309.

Sofroniew, N.J., Flickinger, D., King, J., Svoboda, K., 2016. A large field of view twophoton mesoscope with subcellular resolution for in vivo imaging. Elife 5, 5.

Szalay, G., Judák, L., Katona, G., Ócsai, K., Juhász, G., Veress, M., Szadai, Z., Fehér, A., Tompa, T., Chiovini, B., Maák, P., Rózsa, B., 2016. Fast 3D imaging of spine, dendritic, and neuronal assemblies in behaving animals. Neuron 92, 723-738.

Tan, Z., Sun, W., Chen, T.-W., Kim, D., Ji, N., 2015. Neuronal representation of ultraviolet visual stimuli in mouse primary visual cortex. Sci. Rep. 5, 12597.
Tikidji-Hamburyan, A., Reinhard, K., Storchi, R., Dietter, J., Seitter, H., Davis, K.E., Idrees, S., Mutter, M., Walmsley, L., Bedford, R.A., Ueffing, M., Ala-Laurila, P., Brown, T.M., Lucas, R.J., Münch, T.A., 2017. Rods progressively escape saturation to drive visual responses in daylight conditions. Nat. Commun. 8, 1813.

Umino, Y., Solessio, E., Barlow, R.B., 2008. Speed, spatial, and temporal tuning of rod and cone vision in mouse. J. Neurosci. 28, 189-198.

Whitney, I.E., Raven, M.A., Lu, L., Williams, R.W., Reese, B.E.A., 2011. QTL on chromosome 10 modulates cone photoreceptor number in the mouse retina. Invest. Ophthalmol. Vis. Sci. 52, 3228-3236.

Yang, W., Carrillo-Reid, L., Bando, Y., Peterka, D.S., Yuste, R., 2018. Simultaneous twophoton imaging and two-photon optogenetics of cortical circuits in three dimensions. eLife 7, e32671. 